\title{
Factores asociados a calidad de vida relacionada con la salud de pacientes trasplantados de riñón
}

\author{
Carolina Pérez Blancas, $\mathbf{M}^{\mathrm{a}}$ Carmen Moyano Espadero, Miriam Estepa del Árbol, Rodolfo Crespo Montero \\ Facultad de Medicina y Enfermería de Córdoba, Hospital Universitario Reina Sofía. Córdoba. España
}

\section{Resumen}

Introducción: El Trasplante Renal es la terapia renal sustitutiva que proporciona mayores beneficios a los pacientes con Insuficiencia Renal Crónica Terminal. Aún así, el paciente que ha recibido un injerto renal, sigue siendo un enfermo crónico que no tiene resueltos sus problemas de salud y cuya calidad de vida está asociada con diferentes factores que pueden influir en la misma.

Objetivo: Conocer la producción científica sobre la CVRS del paciente TR.

Metodología: Se realizó una búsqueda en las bases de datos de Pubmed, Science Direct, Google Académico, Scielo y Scopus. Se incluyeron artículos científicos escritos en inglés y español. Posteriormente se analizaron los artículos que trataban sobre la calidad de vida en el paciente trasplantado renal, excluyendo aquellos artículos que no presentaban resultados o no aportaban suficientes datos y aquellos que tenían como objeto de estudio pacientes pediátricos.

Resultados: Se han incluido en esta revisión 42 artículos publicados entre el año 2000 y 2015. La mayoría de los estudios son trasversales y observacionales descriptivos y el Short Form-36 Health Survey es el instrumento más usado. Las variables estudiadas han sido género, edad, estado civil, nivel de estudios, datos bioquímicos, empleo, ingresos, tratamiento inmunosupresor, problemas psicológicos, diagnóstico principal, tiempo post TR, tiempo en diálisis, trasplante vivo vs cadáver y comorbilidades. Los datos que han mostra-

Correspondencia:
Carolina Pérez Blancas
Avda. de la Salud, 20 B. 14840 Castro del Río. Córdoba
E-mail: n12peblc@uco.es

do correlación significativa sobre CVRS han sido género, estado civil, ingresos, nivel de estudios, datos bioquímicos, tratamiento, tiempo en diálisis y comorbilidades.

Conclusiones: A la vista de estos resultados podemos afirmar que los factores que influyen de manera positiva sobre la CVRS son el sexo masculino, estar casado o tener pareja, alto nivel de estudios, niveles elevados de hemoglobina y hematocrito, y trasplante renal procedente de donante vivo. EI TR presenta mejor CVRS que los pacientes en HD y DP, pero peor que la de la población general. Estos resultados se muestran independientes del instrumento usado para medir la calidad de vida relacionada con la salud.

\section{PALABRAS CLAVE}

- CALIDAD DE VIDA

- TRASPLANTE RENAL

Factors associated with health-related quality of life in renal transplant recipients

Abstract

Introduction: Kidney Transplantation is the renal replacement therapy that provides greater benefits to patients with chronic renal failure in the end stage. Even so, the patient has received a kidney transplant, it remains a chronic sick that has not solved their health problems and whose quality of life is associated with different factors that can influence it.

Objective: Knowing the bibliographic production on HRQOL TR patient. 
Methods: A search was conducted in the databases of de Pubmed, Science Direct, Google Scholar, Scielo and Scopus. Scientific articles written in English and Spanish were included. Later articles dealing with the quality of life in renal transplant patients were analyzed, excluding those items which do not have or do not show sufficient results data and those that were intended to study pediatric patients.

Results: It has included in this review 42 articles published between 2000 and 2015. Most of the studies are observational and descriptive transversal and the Short Form-36 Health Survey is the instrument used. The variables studied were gender, age, marital status, education level, biochemical data, employment, income, immunosuppressive therapy, psychological problems, principal diagnosis, time post $\mathrm{KT}$, time on dialysis, transplant alive vs dead and comorbidities. The data have shown significant correlation on HRQOL were gender, marital status, income, educational level, biochemical data, treatment, time on dialysis and comorbidities.

Conclusions: In view of these results we can say that the factors that influence positively on HRQOL include male gender, being married or having a partner, highly educated, high levels of hemoglobin and hematocrit, and kidney transplant from a living donor. The KT has better HRQL than patients on HD and PD, but worse than that of the general population. These results are independent of the instrument used to measure the quality of life related to health.

\section{KEYWORDS}

\section{- QUALITY OF LIFE \\ - KIDNEY TRANSPLANT}

\section{Introducción}

La enfermedad renal crónica (ERC) constituye hoy día, un importante problema de salud pública. La manifestación más avanzada de la ERC, la insuficiencia renal crónica terminal (IRCT) y la consiguiente necesidad de tratamiento sustitutivo de la función renal mediante diálisis peritoneal (DP) o trasplante renal (TR), presenta una incidencia y una prevalencia crecientes en las últimas décadas ${ }^{1-5}$.

En efecto, la prevalencia de la ERC ha aumentado progresivamente y los registros de todos los países que poseen datos muestran un continuo crecimiento de pa- cientes que necesitan tratamiento sustitutivo de la función renal (TRS) ${ }^{6}$, tal como muestran los registros de la Sociedad Española de Nefrología y de la European Dialysis and Transplant Association ${ }^{7,8}$.

EI TR es el tratamiento de elección para la IRCT, siendo su principal objetivo el logro de la calidad y cantidad de vida máxima y reducir al mínimo los efectos de la enfermedad y los costos de la atención en este proceso, ya que estos costos no se limitan sólo al procedimiento de trasplante sino también a los gastos que surgen en el tratamiento de los eventos adversos, algunos de ellos provocados por el tratamiento inmunosupresor ${ }^{9,10}$.

España ha alcanzado la tasa más elevada del mundo en donación y trasplantes por millón de habitantes frente, a lo que en la década de los setenta del siglo pasado era un reto lejano ${ }^{11}$. Esto ha sido posible, gracias a los avances en los procedimientos de TR y terapias inmunosupresoras, que han permitido aumentar notablemente, las tasas de supervivencia del injerto, que a un año son actualmente de más del $90 \%{ }^{12}$. En nuestro país, un $51.7 \%$ de los pacientes que vive con TRS lo hacen mediante TR funcionante, lo que ha llevado a España a una posición de liderazgo con respecto al trasplante de este órgano sólido ${ }^{7}$.

Cabe señalar que entre las causas de mayor supervivencia de los injertos renales destacan aquellos injertos procedentes de donante vivo. Un factor que ha demostrado la gran influencia de ello de manera independiente es la ausencia de diálisis o el menor tiempo en esta terapia del receptor, que hace que esté en mejores condiciones para recibir un trasplante. Además en estos casos se obtienen mejores cifras de función renal y menos morbilidad asociada a TR ${ }^{13}$.

No obstante, aún siendo el mejor procedimiento de TRS, el trasplante no está exento de complicaciones a medio y largo plazo, aunque se ha comunicado una mejor percepción de la Calidad de Vida Relacionada con la Salud (CVRS) en comparación con la HD y la DP'14.

Es por ello, por lo que el interés por el análisis de la CVRS en pacientes con TR, ha aumentado en los últimos años, pues teóricamente, este tratamiento es el que consigue mejores niveles de calidad de vida y la mejor rehabilitación para el paciente con IRCT ${ }^{15-17}$. Además, la calidad de vida puede referirse no sólo a la pérdida de salud a causa de una determinada enfermedad, como lo es la IRCT, sino también a la situación vital que se genera tras haber recibido un tratamiento médico como es el TR. Ambos están íntimamente rela- 
cionados, puesto que la percepción de la calidad de vida tras el tratamiento tiene mucho que ver con la percibida anteriormente durante el periodo de enfermedad. El paciente trasplantado "no es un paciente libre de morbilidad ya que continúa necesitando un control médico especializado y frecuente" además de estar sometido continuamente a un tratamiento inmunosupresor que puede provocar efecto secundarios y llevar a una serie de complicaciones médicas que son habituales en él, por lo que aunque haya sido sometido a TRS continua manteniendo la característica de paciente crónico ${ }^{18}$.

Por lo tanto, es importante incluir indicadores de calidad de vida en el seguimiento de estos pacientes, debido a la estrecha relación entre calidad de vida, morbilidad y mortalidad ${ }^{19}$.

Existen muchos factores que inciden en la calidad de vida de este tipo de pacientes y que permiten realizar comparaciones entre ellos, con el fin de determinar cuáles influyen de manera directa e indirecta en la mejora de CVRS tras la realización de TR. La edad, sexo, nivel de educación, nivel económico, diagnóstico principal de enfermedad, tiempo en terapia previa con DP o HD, tiempo de evolución del trasplante, número de días de hospitalización, índice de comorbilidad, cumplimiento del tratamiento inmunosupresor, injerto procedente de donante vivo o donante cadáver, son algunos de los factores que pueden influir en la CVRS de los pacientes con TR tanto en la dimensión física como en la dimensión psicosocia| ${ }^{20,21}$.

El grado de mejoría de la CVRS varía en los diversos pacientes sometidos a la misma terapia. Esta variabilidad se explica sobre todo por los factores que afectan a CVRS después del TR, entre las cuales las variables sociodemográficas y médicas son las más frecuentemente investigadas. Existen además estudios que han investigado acerca de las variables psicosociales confirmando que el afecto negativo, rasgos de personalidad y la angustia intervienen en la percepción de los síntomas y la estimación de la CVRS de estos pacientes ${ }^{22}$.

Teniendo en cuenta que la percepción de la calidad de vida del paciente con TR está íntimamente ligada a la percepción que tenían esos pacientes en su situación de IRCT con tratamiento sustitutivo dialítico, no es extraño que los pacientes después de ser trasplantados conceptualicen la calidad de vida como la calidad de regresar a la vida normal, al reincorporarse a actividades que antes no podían hacer por las complicaciones propias de la IRC. Entre las razones que podían explicar una peor CVRS antes de TR, en la fase de lista de espera, podemos hacer referencia al deterioro físico, cansancio emocional DP ó HD, pérdida de libertad al asistir continuamente al centro asistencial ${ }^{23,24}$. Respecto a las expectativas de los pacientes en lista de espera para TR son más altas que la calidad de vida que realmente tendrán ya que a veces los pacientes son demasiado optimistas respecto al trasplante si tenemos en cuenta los efectos secundarios de los esteroides y que los perciben como muy perturbadores llegando incluso a limitar la mejoría que produce el trasplante ${ }^{24,25}$.

Por todo ello, y teniendo en cuenta que el paciente con TR va a vivir muchos años con un órgano trasplantado pero con bastantes inconvenientes derivados del tratamiento inmunosupresor, $y$ que en el fondo sigue siendo un paciente con una patología crónica que no tiene resueltos todos sus problemas de salud, es por lo que hemos decidido estudiar una de las variables más importantes en la vida del paciente TR, como es la calidad de vida.

\section{Objetivos}

\section{General:}

- Conocer la producción científica sobre la CVRS del paciente con TR.

\section{Específicos:}

1. Conocer los factores influyentes en la CVRS del paciente TR.

2. Determinar los cambios en la CVRS después del TR.

\section{Metodología}

El diseño empleado es el de un estudio de revisión sistemática. Para ello, se realizó una búsqueda en diversas bases de datos con el objetivo de recopilar la mayor producción posible disponible en relación con el tema a tratar.

Las bases de datos empleadas fueron: Pubmed, Science Direct, Google Académico, Scielo y Scopus. El periodo de consulta de bases de datos tuvo lugar desde 17 febrero de 2015 hasta el 30 de abril de 2015.

Las palabras clave que fueron utilizadas en las diferentes bases de datos fueron: calidad de vida (quality of life) y trasplante renal (kidney transplant). Teniendo en cuenta que los distintos descriptores empleados estuvieran presente en el resumen, título del artículo $y$, si la base de datos lo presenta, en palabras clave. 
Los criterios de inclusión fueron:

- Estudios de investigación en los que se tratara la calidad de vida de los pacientes trasplantados de riñón, sus factores asociados y cambios en la calidad de vida antes y después del TR.

- Artículos originales y revisiones sistemáticas.

- Individuos adultos en cualquier rango de edad.

- Estudios que tuvieran como resultado haber evaluado la calidad de vida del paciente adulto con $T R$, ya sea exclusivo o en asociación con otras variables.

Los criterios de exclusión fueron:

- Artículos que no presentaban resultados.

- Solamente se aceptaron artículos en inglés y en castellano, puesto que son las dos lenguas con mayor producción científica y que el revisor conoce.

Al inicio de la búsqueda no se tuvieron en cuenta los criterios de inclusión y exclusión en las diversas bases de datos, para así conocer la cantidad de producción científica existente en el tema y obtener mayor conocimiento del mismo, de manera que se aceptaron todos los trabajos que tratasen sobre el tema en cuestión. Posteriormente, se colocaron los criterios de inclusión/exclusión en las bases de datos y se siguió la estrategia de búsqueda según la figura 1 .
Diversas bases de datos no permitían introducir como criterios de exclusión el lenguaje del artículo ni permitían solo obtener artículos científicos y revisiones (no excluían cartas al director, guías prácticas...). Una vez obtenida la producción científica empleando los descriptores en las bases de datos con los distintos métodos de búsqueda, los artículos extraídos se recopilaron en una carpeta.

Tras la lectura de todos los títulos y resúmenes de los artículos encontrados se fueron descartando aquellos que no cumplían con los criterios de inclusión y exclusión. Posteriormente se realizó la lectura completa de los trabajos seleccionados y se realizó un nuevo descarte de aquellos que no versaban exactamente sobre el tema a estudiar.

Finalmente el número de artículos total que cumplían con los criterios de inclusión y que se han utilizado para el estudio ha sido de 42. En ese momento se comenzó el análisis de los artículos recopilados.

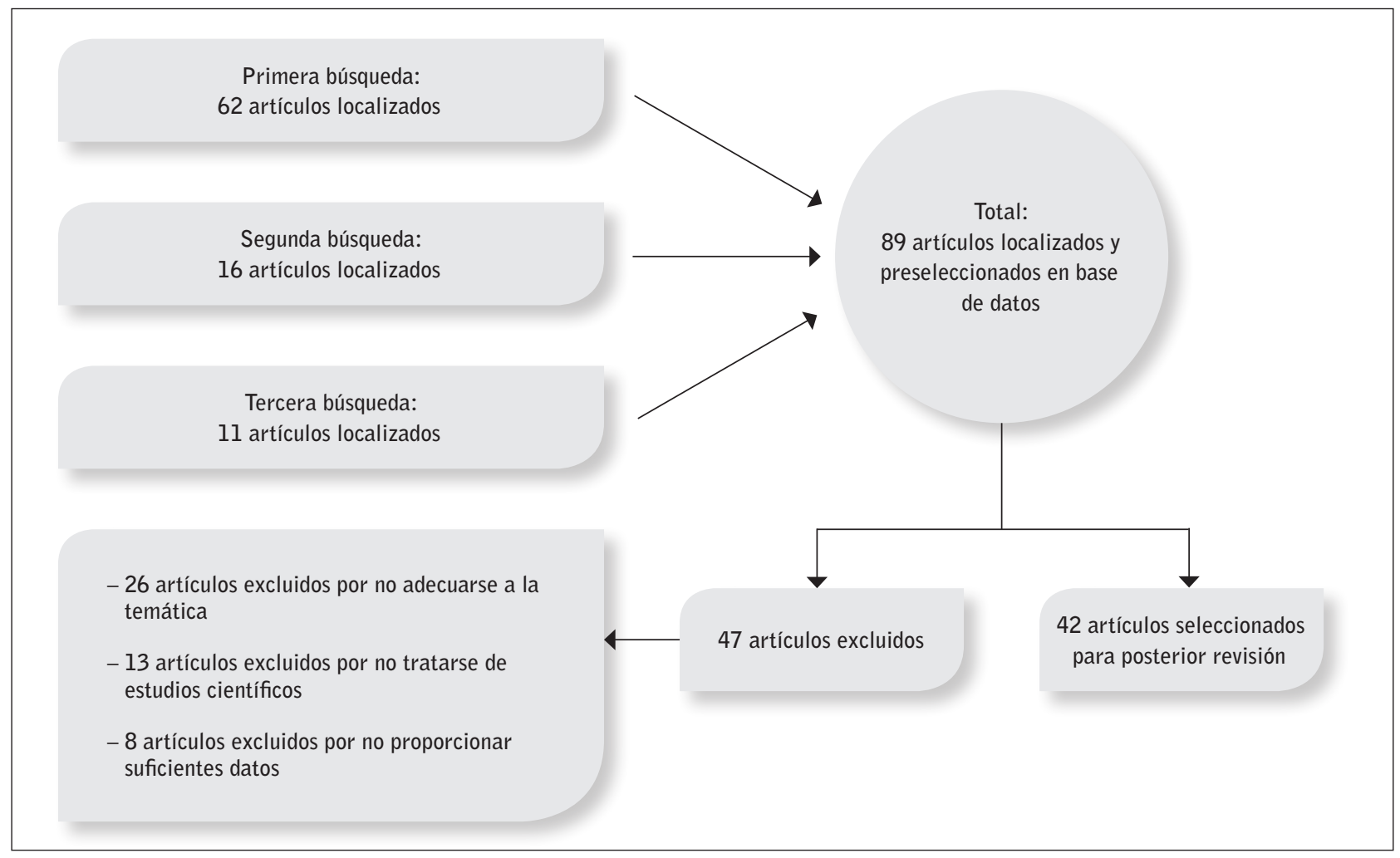

Figura 1. 


\section{Resultados}

\section{Selección de estudios preliminar}

En total, de los 89 documentos preseleccionados de todas las bases de datos, tras eliminar 47 , se quedaron 42 artículos que cumplían con los criterios de inclusión/exclusión establecidos en este estudio de revisión, tal como puede observarse en la tabla I.

\section{Análisis de los resultados}

Las variables analizadas en cada uno de los artículos seleccionados para revisión, así como los datos más relevantes de cada estudio, que han sido clave para la obtención de los resultados, se muestran en la tabla II.
Tabla 1. Resultados de la búsqueda inicial

\begin{tabular}{|l|c|c|c|c|c}
\hline Bases de datos & PubMed & $\begin{array}{c}\text { Science } \\
\text { Direct }\end{array}$ & $\begin{array}{c}\text { Google } \\
\text { Académico }\end{array}$ & Scielo & Scopus \\
\hline $\begin{array}{l}\text { Total de } \\
\text { publicaciones }\end{array}$ & 25 & 27 & 14 & 18 & 5 \\
\hline $\begin{array}{l}\text { localizadas } \\
\begin{array}{l}\text { Total de } \\
\text { publicaciones } \\
\text { seleccionadas }\end{array}\end{array}$ & 12 & 13 & 6 & 9 & 2 \\
\hline
\end{tabular}

Tabla II. Características de los cuidadores

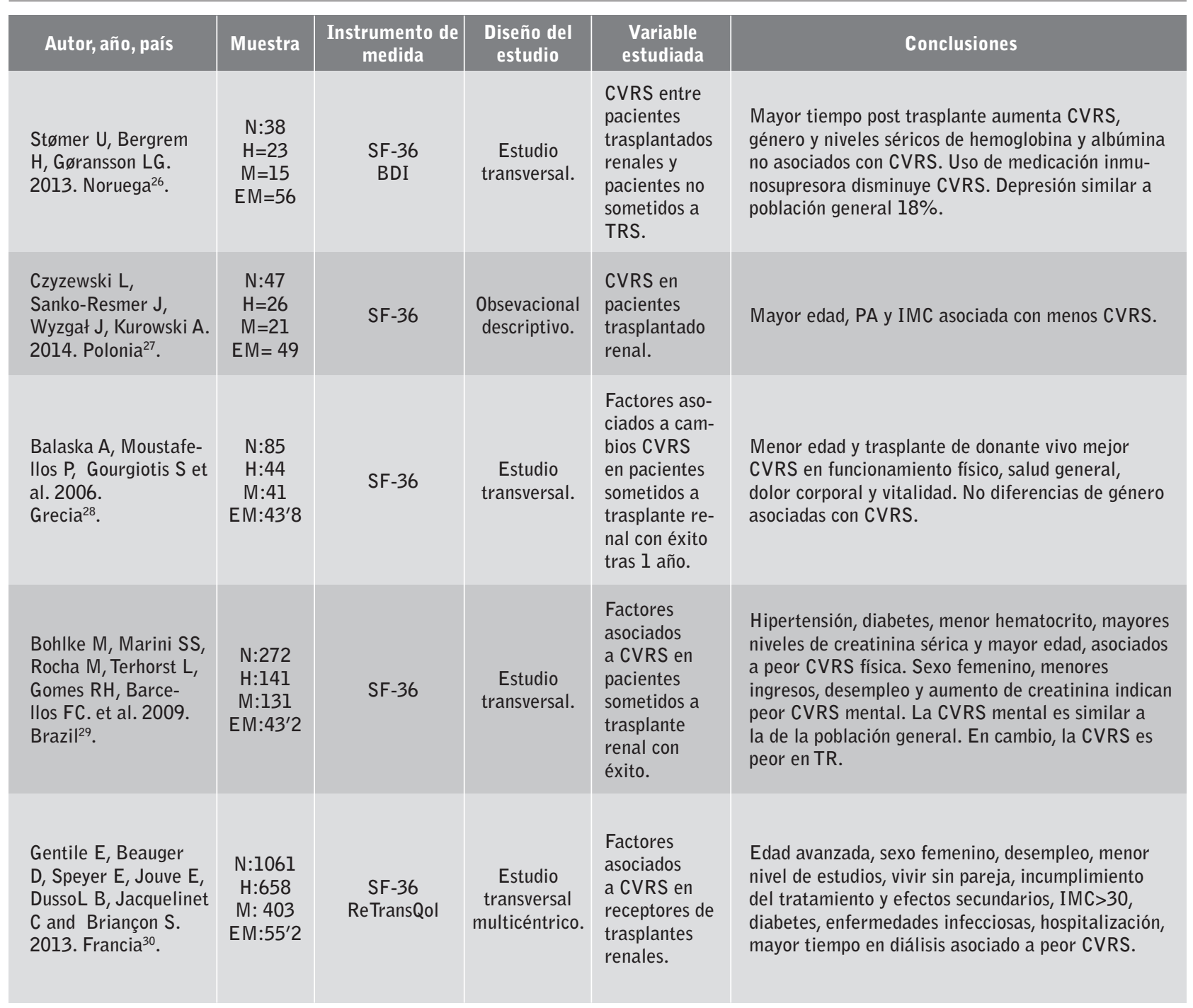




\begin{tabular}{|c|c|c|c|c|c|}
\hline $\begin{array}{l}\text { Rosenberger J, van } \\
\text { Dijk J.P, Nagyova I, } \\
\text { Roland R, Madaraso- } \\
\text { va Geckova A, J. A. } \\
\text { van den Heuvel W and } \\
\text { W. Groothoff J. } 2005 \text {. } \\
\text { Paises bajos }{ }^{31} \text {. }\end{array}$ & $\begin{array}{c}N: 128 \\
H: 86 \\
M: 42 \\
E M: 48^{\prime} 6\end{array}$ & SF-36 & $\begin{array}{l}\text { Estudio } \\
\text { observacional } \\
\text { descriptivo. }\end{array}$ & $\begin{array}{l}\text { Factores } \\
\text { médicos rela- } \\
\text { cionados con } \\
\text { el trasplante } \\
\text { renal que } \\
\text { afectan al es- } \\
\text { tado de salud } \\
\text { percibido. }\end{array}$ & $\begin{array}{l}\text { Edad avanzada, menor educación, sexo femenino, } \\
\text { mayor tiempo en diálisis, diabetes, elevados niveles } \\
\text { de creatinina en pacientes más jóvenes, se asocia } \\
\text { con peor CVRS. }\end{array}$ \\
\hline $\begin{array}{l}\text { Arogundade F A, } \\
\text { Abd-Essamie M A, } \\
\text { Barsoum R S. } 2005 . \\
\text { Egypto }^{32} \text {. }\end{array}$ & $\begin{array}{c}N: 52 \\
H: 31 \\
M: 21 \\
E M: 38^{\prime} 23\end{array}$ & $\begin{array}{l}\text { Escala de estado } \\
\text { funcional de } \\
\text { Karnofsky }\end{array}$ & $\begin{array}{l}\text { Estudio } \\
\text { observacional } \\
\text { descriptivo. }\end{array}$ & $\begin{array}{l}\text { CVRS en } \\
\text { pacientes } \\
\text { trasplantados } \\
\text { renales. }\end{array}$ & $\begin{array}{l}\text { Mayor edad, sexo femenino, mayores niveles de } \\
\text { creatinina sérica, urea, fosfato en suero, menores } \\
\text { niveles de hemoglobina y ser viuda o divorciada se } \\
\text { asocia con peor CVRS. }\end{array}$ \\
\hline $\begin{array}{l}\text { Wei TY, Chiang YJ, } \\
\text { Hsieh CY, Weng LC, } \\
\text { Lin SC, Lin MH. } \\
\text { 2013. Taiwan } 33 \text {. }\end{array}$ & $\begin{array}{c}\mathrm{N}: 88 \\
\mathrm{H}: 40 \\
\mathrm{M}: 44 \\
\mathrm{EM}: 49^{\prime} 1\end{array}$ & MOS SF-36 & $\begin{array}{l}\text { Diseño } \\
\text { transversal y } \\
\text { correlacional. }\end{array}$ & $\begin{array}{l}\text { CVRS a } \\
\text { largo plazo } \\
\text { en pacientes } \\
\text { con trasplante } \\
\text { renal. }\end{array}$ & $\begin{array}{l}\text { TR a largo plazo junto con sexo femenino y mayor } \\
\text { edad, está asociado a peor CVRS que la población } \\
\text { general en las subescalas de: funcionamiento físico, } \\
\text { salud general, vitalidad, funcionamiento social y } \\
\text { salud mental. Dolor corporal similar al de la pobla- } \\
\text { ción general. Menores nivel de creatinina sérica y } \\
\text { empleo mejor puntuación en CVRS. }\end{array}$ \\
\hline $\begin{array}{l}\text { Wei TY, Chiang YJ, } \\
\text { Hsieh CY, Weng LC, } \\
\text { Lin SC, Lin MH. } \\
\text { 2013. Taiwan'33. }\end{array}$ & $\begin{array}{c}\mathrm{N}: 88 \\
\mathrm{H}: 40 \\
\mathrm{M}: 44 \\
\mathrm{EM}: 49^{\prime} 1\end{array}$ & MOS SF-36 & $\begin{array}{c}\text { Diseño } \\
\text { transversal y } \\
\text { correlacional. }\end{array}$ & $\begin{array}{l}\text { CVRS a } \\
\text { largo plazo } \\
\text { en pacientes } \\
\text { con trasplante } \\
\text { renal. }\end{array}$ & $\begin{array}{l}\text { TR a largo plazo junto con sexo femenino y mayor } \\
\text { edad, está asociado a peor CVRS que la población } \\
\text { general en las subescalas de: funcionamiento físico, } \\
\text { salud general, vitalidad, funcionamiento social y } \\
\text { salud mental. Dolor corporal similar al de la pobla- } \\
\text { ción general. Menores nivel de creatinina sérica y } \\
\text { empleo mejor puntuación en CVRS. }\end{array}$ \\
\hline $\begin{array}{l}\text { Mendonça Ana Elza } \\
\text { Oliveira de, Salvetti } \\
\text { Marina de Góes, Maia } \\
\text { Eulália Maria Chaves } \\
\text { et } 2015 \text {. Brazil }{ }^{34} \text {. }\end{array}$ & $\begin{array}{c}N: 63 \\
H: 39 \\
M: 24 \\
E M: 39^{\prime} 9\end{array}$ & WHOQOL-Bref & $\begin{array}{c}\text { Estudio } \\
\text { longitudinal. }\end{array}$ & $\begin{array}{l}\text { Aspectos } \\
\text { físicos de la } \\
\text { CVRS en } \\
\text { los pacientes } \\
\text { receptores de } \\
\text { riñón. }\end{array}$ & $\begin{array}{l}\text { EI TR aumenta las puntuaciones de dominio físico } \\
\text { de la CVRS frente a la etapa pre-trasplante: dolor, } \\
\text { sueño, fatiga, menor dependencia de tratamiento, } \\
\text { mejor capacidad para moverse y trabajar. }\end{array}$ \\
\hline $\begin{array}{l}\text { Griva K, Stygall J, } \\
\text { Hui Ng JH, Da- } \\
\text { venport A, Harrison } \\
\text { MJ, and Newman S. } \\
\text { 2011. Singapore } 35 \text {. }\end{array}$ & $\begin{array}{c}N: 102 \\
E M: 46^{\prime} 57\end{array}$ & $\begin{array}{l}\text { SF-36 } \\
\text { Escala de estado } \\
\text { funcional de } \\
\text { Karnofsky }\end{array}$ & $\begin{array}{l}\text { Estudio de } \\
\text { cohortes } \\
\text { prospectivo. }\end{array}$ & $\begin{array}{l}\text { CVRS tras } \\
6 \text { años } \\
\text { post-tras- } \\
\text { plante renal } \\
\text { asociada a } \\
\text { cambios en } \\
\text { aspecto físico, } \\
\text { social, mental } \\
\text { y emocional. }\end{array}$ & $\begin{array}{l}\text { Desde el inicio hasta los } 6 \text { años post trasplante, la } \\
\text { CVRS emocional mejora y la CVRS física disminu- } \\
\text { ye. Injerto procedente de donante vivo mejora el } \\
\text { funcionamiento físico pero provoca mayor deterioro. } \\
\text { Mayor edad asociada con menor preocupación } \\
\text { respecto al trasplante. }\end{array}$ \\
\hline $\begin{array}{l}\text { Ocampo Otálvaro LE, } \\
\text { Zapata Rueda CM, } \\
\text { Villa Londoño AJ y } \\
\text { Vinaccia Alpi E. } \\
\text { 2007. Colombia }{ }^{36} \text {. }\end{array}$ & $\begin{array}{c}N: 100 \\
H: 46 \\
M: 54 \\
E M: 42^{\prime} 25\end{array}$ & WH0Q0L-100 & $\begin{array}{c}\text { Análisis } \\
\text { descriptivo. }\end{array}$ & $\begin{array}{l}\text { CVRS en } \\
\text { pacientes } \\
\text { trasplantados } \\
\text { de riñón, híga- } \\
\text { do y médula } \\
\text { ósea. Varia- } \\
\text { bles socio } \\
\text { demográficas } \\
\text { y psicológicas. }\end{array}$ & $\begin{array}{l}\text { Estado civil: casado se asocia con mayor CVRS. EI } \\
\text { TR aumenta las puntuaciones de todos los dominios } \\
\text { salvo las del dolor y disconfort, sueño y descanso, y } \\
\text { afecto negativo. }\end{array}$ \\
\hline $\begin{array}{l}\text { Ortega T, Deulofeu } \\
\text { R, Salamero P, et al. } \\
\text { 2009. España }{ }^{37} \text {. }\end{array}$ & $\begin{array}{l}N: 126 \\
H: 85 \\
M: 41 \\
E M: 53\end{array}$ & SF-36 & $\begin{array}{c}\text { Estudio } \\
\text { prospectivo. }\end{array}$ & $\begin{array}{l}\text { CVRS antes } \\
\text { y después del } \\
\text { trasplante } \\
\text { renal. }\end{array}$ & $\begin{array}{l}\text { Mejora de la salud mental a los } 12 \text { meses frente a } \\
\text { los } 3 \text { meses post trasplante. Mayor CVRS asociada } \\
\text { con mayor edad y sexo femenino igual o superior } \\
\text { que el masculino. }\end{array}$ \\
\hline
\end{tabular}


[ Carolina Pérez Blancas, et al ]

Factores asociados a la calidad de vida relacionada con la salud de pacientes trasplantados de riñón

\begin{tabular}{|c|c|c|c|c|c|}
\hline $\begin{array}{l}\text { Rodríguez Martín } \\
M^{\mathrm{a} C} \text {, Conejos Alegre } \\
\text { C, Fuenmayor Díaz } \\
\text { A, et al. } \\
\text { 2009. España }{ }^{18} \text {. }\end{array}$ & $\begin{array}{c}\mathrm{N}: 31 \\
\mathrm{H}: 14 \\
\mathrm{M}: 17 \\
\mathrm{EM}: 71^{\prime} 8\end{array}$ & SF-36 & $\begin{array}{c}\text { Estudio } \\
\text { observacional } \\
\text { descriptivo. }\end{array}$ & $\begin{array}{l}\text { CVRS en } \\
\text { pacientes } \\
\text { mayores de } \\
65 \text { años. }\end{array}$ & $\begin{array}{l}\text { Edad no asociada a CVRS. Mejora de la percepción } \\
\text { de CVRS TR respecto al periodo anterior. Mayor } \\
\text { ampliación de número de actividades a realizar, } \\
\text { salvo en aquellas actividades que requieren mayor } \\
\text { esfuerzo físico. }\end{array}$ \\
\hline $\begin{array}{l}\text { Canché-Arenas AP, } \\
\text { Reza-Orozco M, } \\
\text { Rodríguez-Weber FL. } \\
\text { 2011. México }{ }^{38} \text {. }\end{array}$ & $\begin{array}{l}\mathrm{N}: 30 \\
\mathrm{H}: 19 \\
\mathrm{M}: 11 \\
\mathrm{EM}: 49\end{array}$ & $\begin{array}{l}\text { Escala } \\
\text { Karnofsky }\end{array}$ & $\begin{array}{c}\text { Estudio } \\
\text { descriptivo y } \\
\text { retrospectivo. }\end{array}$ & $\begin{array}{l}\text { CVRS en } \\
\text { pacientes con } \\
\text { trasplante } \\
\text { renal. Edad, } \\
\text { trasplante } \\
\text { vivo vs cadá- } \\
\text { ver, factores } \\
\text { médicos y } \\
\text { sociales. }\end{array}$ & $\begin{array}{l}\text { Mejora de la CVRS en todas las dimensiones de la } \\
\text { escala de Karnofsky con una diferencia de } 20 \text { puntos } \\
\text { respecto al periodo pre trasplante. Pese a ello, se } \\
\text { mantiene la dificultad para realizar trabajo activo. }\end{array}$ \\
\hline $\begin{array}{l}\text { Rebollo P, Bobes J, } \\
\text { González M.P, Saiz } \\
\text { P, Ortega F. } 2000 . \\
\text { España }^{39} \text {. }\end{array}$ & $\begin{array}{l}\mathrm{N}: 210 \\
\mathrm{H}: 140 \\
\mathrm{M}: 70 \\
\mathrm{EM}: 51\end{array}$ & SF-36 & $\begin{array}{l}\text { Estudio } \\
\text { transversal. }\end{array}$ & $\begin{array}{l}\text { CVRS } \\
\text { asociadas a } \\
\text { variables so- } \\
\text { cio- demográ- } \\
\text { ficas y clínicas } \\
\text { en pacientes } \\
\text { con TRS. }\end{array}$ & $\begin{array}{l}\text { Mayor CVRS física se asocia con edad avanzada, } \\
\text { menor tiempo en diálisis y menor índice de mortali- } \\
\text { dad, y mayor CVRS mental se asocia con más edad } \\
\text { y menor tiempo post trasplante. }\end{array}$ \\
\hline $\begin{array}{l}\text { Helga Franke G, } \\
\text { Heemann U, Kohnle } \\
\text { M, Luetkes P, Mae- } \\
\text { hner N, Reimer J. } \\
2000^{40} \text {. }\end{array}$ & $\begin{array}{l}N: 149 \\
H: 92 \\
M: 57 \\
\text { EM: } 20 \\
>60\end{array}$ & $\begin{array}{l}\text { MLDL } \\
\text { BSI } \\
\text { K-22 }\end{array}$ & $\begin{array}{c}\text { Estudio } \\
\text { observacional } \\
\text { descriptivo. }\end{array}$ & $\begin{array}{l}\text { CVRS antes } \\
\text { y después del } \\
\text { trasplante, } \\
\text { en pacientes } \\
\text { con trasplante } \\
\text { renal. }\end{array}$ & $\begin{array}{l}\text { Mayor CVRS asociada a mayor edad en la } \\
\text { dimensión psicológica debido a mayor estabilidad } \\
\text { psicológica. Respecto a la dimensión física mayor } \\
\text { CVRS para los jóvenes, lo que va asociada a mayor } \\
\text { supervivencia. }\end{array}$ \\
\hline $\begin{array}{l}\text { Tomasz W, Piotr S. } \\
\text { 2003. Polonia }{ }^{41} \text {. }\end{array}$ & $\begin{array}{c}N: 83 \\
H: 43 \\
M: 40 \\
E M: 43^{\prime} 30\end{array}$ & WH0QOL-100 & $\begin{array}{c}\text { Estudio } \\
\text { observacional } \\
\text { descriptivo. }\end{array}$ & $\begin{array}{l}\text { CVRS en } \\
\text { paciente } \\
\text { trasplantado } \\
\text { renal. }\end{array}$ & $\begin{array}{l}\text { CVRS mejora en los dominios: físico, social, medio } \\
\text { ambiente, dolor, energía, sentimientos positivos, } \\
\text { movilidad, ABVD, capacidad de trabajo, actividad } \\
\text { sexual y transporte en los pacientes trasplantados } \\
\text { respecto a los sometidos a HD. La imagen corporal y } \\
\text { el aspecto de la CVRS es peor en trasplantados. }\end{array}$ \\
\hline $\begin{array}{l}\text { Acosta Hernández } \\
\text { PA, Chaparro López } \\
\text { LC, Rey Anaconda } \\
\text { CA. 2008. Colombia } 3 \text {. }\end{array}$ & $\begin{array}{c}N: 40 \\
H: 25 \\
M: 15 \\
E M: 45\end{array}$ & WH0Q0L-100 & $\begin{array}{c}\text { Estudio } \\
\text { comparativo. }\end{array}$ & $\begin{array}{l}\text { CVRS en } \\
\text { pacientes con } \\
\text { trasplante re- } \\
\text { nal. Variables } \\
\text { de estrategia } \\
\text { de afronta- } \\
\text { miento y CV. } \\
\text { Factores socio } \\
\text { demográficos. }\end{array}$ & $\begin{array}{l}\text { Pacientes casados y con estudios universitarios pun- } \\
\text { tuaciones más altas en la mayoría de las variables } \\
\text { de afrontamiento y CVRS. Mujeres nivel superior } \\
\text { en salud física, psicológica, relaciones sociales } \\
\text { espiritualidad y calidad global. Pacientes TR y con } \\
\text { mayor rango de edad mejores puntuaciones que } \\
\text { pacientes en HD. }\end{array}$ \\
\hline $\begin{array}{l}\text { Borroto Díaz G, } \\
\text { Almeida Fernández } \\
\text { J, Lorenzo Clemente } \\
\text { A, Alfonso Sat F, Gue- } \\
\text { rreo Díaz C. } 2007 . \\
\text { Cuba. }{ }^{14} \text {. }\end{array}$ & $\begin{array}{l}N: 32 \\
H: 19 \\
M: 13 \\
E M: 41\end{array}$ & SF-36 & $\begin{array}{l}\text { Estudio } \\
\text { prospectivo, } \\
\text { exploratorio y } \\
\text { descriptivo. }\end{array}$ & $\begin{array}{l}\text { Percepción } \\
\text { CVRS en } \\
\text { enfermos } \\
\text { sometidos a } \\
\text { HD oTR. }\end{array}$ & $\begin{array}{l}\text { Mayor CVRS asociada a sexo masculino, más edad, } \\
\text { menor número de hospitalizaciones, y niveles de } \\
\text { albumina sérica y hemoglobina superiores a } 34 \mathrm{mg} / \\
\text { dl y } 1 l^{\prime} 6 \text { respectivamente. El apoyo familiar es el } \\
\text { parámetro de más importancia. }\end{array}$ \\
\hline $\begin{array}{l}\text { Rebollo P, González } \\
\text { M.P, Bobes J, Saiz } \\
\text { P, Ortega F. } 2000 . \\
\text { España }^{16} \text {. }\end{array}$ & $\begin{array}{c}N: 210 \\
H: 141 \\
M: 69 \\
E M: 47^{\prime 2} 2 \\
9-67^{\prime} 58\end{array}$ & SF-36 & $\begin{array}{l}\text { Estudio } \\
\text { transversal. }\end{array}$ & $\begin{array}{l}\text { CVRS en } \\
\text { pacientes en } \\
\text { TRS. Tras- } \\
\text { plante renal. }\end{array}$ & $\begin{array}{l}\text { No se asocia diferencia significativa entre la edad y } \\
\text { CVRS. EI TR mejora la CVRS en todas las dimen- } \\
\text { siones respecto a los pacientes en HD. }\end{array}$ \\
\hline
\end{tabular}




\begin{tabular}{|c|c|c|c|c|c|}
\hline $\begin{array}{l}\text { Prihodova L, Nagyova } \\
\text { I, Rosenberger J, Ro- } \\
\text { land R, Van Dijk J.P } \\
\text { and Groothoff J.W. } \\
\text { 2009.Paises Bajos } \\
\end{array}$ & $\begin{array}{l}\mathrm{N}: 177 \\
\mathrm{H}: 119 \\
\mathrm{M}: 58 \\
\mathrm{EM}: 48\end{array}$ & SF-36 & $\begin{array}{c}\text { Estudio } \\
\text { observacional. }\end{array}$ & $\begin{array}{l}\text { CVRS física } \\
\text { y mental en } \\
\text { receptores } \\
\text { de trasplante } \\
\text { renal. }\end{array}$ & $\begin{array}{l}\text { Mayor CVRS asociada a menor edad, menor número } \\
\text { de enfermedades comórbidas, menor neuroticismo, } \\
\text { y menor angustia psicológica. Los ingresos y el nivel } \\
\text { de estudios, influyen con correlación positiva sobre } \\
\text { la CVRS. }\end{array}$ \\
\hline $\begin{array}{l}\text { Pérez San Grego- } \\
\text { rio M.A, Martín } \\
\text { Rodríguez A, Galán } \\
\text { Rodríguez A. } 2007 . \\
\text { España }{ }^{26} \text {. }\end{array}$ & $\begin{array}{c}\mathrm{N}: 28 \\
\mathrm{H}: 15 \\
\mathrm{M}: 13 \\
\text { EM: } 40^{\prime} 61\end{array}$ & $\begin{array}{c}\text { SF-36 } \\
\text { EuroQol 5D } \\
\text { ESRD-SCL }\end{array}$ & $\begin{array}{c}\text { Estudio } \\
\text { prospectivo } \\
\text { longitudinal. }\end{array}$ & $\begin{array}{l}\text { Evolución } \\
\text { de CVRS en } \\
\text { pacientes } \\
\text { trasplantados } \\
\text { renales. Fases } \\
\text { pre y post } \\
\text { trasplante. }\end{array}$ & $\begin{array}{l}\text { Los pacientes tras } 1 \text { año del TR presentan mejoría } \\
\text { en todas las dimensiones de los tres cuestionarios en } \\
\text { comparación con la fase pre trasplante, salvo en la } \\
\text { subescala efectos secundarios del tratamiento. }\end{array}$ \\
\hline $\begin{array}{l}\text { Durán Muñoz M.I, } \\
\text { Lope Andrea T, } \\
\text { Del Pino Jurado MåR, } \\
\text { Chicharro Chicharro } \\
M^{a} \text { C, Matilla Villar E. } \\
\text { 2014. España }{ }^{25} \text {. }\end{array}$ & $\begin{array}{c}N: 209 \\
H: 130 \\
M: 79 \\
E M: 55^{\prime} 2\end{array}$ & SF-12 & $\begin{array}{l}\text { Estudio } \\
\text { descriptivo } \\
\text { transversal. }\end{array}$ & $\begin{array}{l}\text { Factores } \\
\text { asociados } \\
\text { a CVRS en } \\
\text { trasplantados } \\
\text { renales. }\end{array}$ & $\begin{array}{l}\text { CVRS del componente físico se asocia con edad me- } \\
\text { nor de } 45 \text { años, mayor nivel de estudios y emplea- } \\
\text { dos. El componente mental presenta asociación con } \\
\text { el sexo masculino y mayor tiempo trasplantado. }\end{array}$ \\
\hline $\begin{array}{l}\text { Maglakelidze } \\
\text { N, Pantsulaia T, } \\
\text { Tchokhonelidze I, } \\
\text { Managadze L, and } \\
\text { Chkhotua A. 2011. } \\
\text { Alemania42. }\end{array}$ & $\mathrm{N}: 48$ & $\begin{array}{l}\text { SF-36 } \\
\text { GBB-24 } \\
\text { Bf-S }\end{array}$ & $\begin{array}{c}\text { Estudio } \\
\text { prospectivo. }\end{array}$ & $\begin{array}{l}\text { CVRS post } \\
\text { trasplante } \\
\text { renal. }\end{array}$ & $\begin{array}{l}\text { Las puntuaciones del TR en el cuestionario SF-36 } \\
\text { y Bf-S no tuvieron diferencias significativas con } \\
\text { respecto al grupo control sano. En todos los compo- } \\
\text { nentes del GBB-24, los receptores de TR anotaron } \\
\text { mayor puntuación que los pacientes en HD y DP. En } \\
\text { fatiga, dolor y quejas cardiacas mostraron peores } \\
\text { puntuaciones que los de DP y sujetos sanos. }\end{array}$ \\
\hline $\begin{array}{l}\text { Aasebø W, Agnete } \\
\text { Homb-Vesteraas } \\
\text { N, Hartmann A and } \\
\text { Stavem K. } 2008 . \\
\text { Noruega }^{43} \text {. }\end{array}$ & $\begin{array}{c}N: 131 \\
H: 58 \\
M: 73 \\
\text { EM: } 29^{\prime} 8\end{array}$ & SF-36 & $\begin{array}{l}\text { Estudio } \\
\text { transversal. }\end{array}$ & $\begin{array}{l}\text { CVRS en } \\
\text { adultos } \\
\text { jóvenes tras- } \\
\text { plantados de } \\
\text { riñón. }\end{array}$ & $\begin{array}{l}\text { Los receptores de TR reportaron altos índices de } \\
\text { participación en actividades deportivas, y la mayoría } \\
\text { estaban satisfechos con su trabajo y vida actual. } \\
\text { Gran parte de los TR de edad 18-35 años se adapta } \\
\text { bien en su vida familiar y profesional. Sin embargo, } \\
\text { en conjunto reportaron menor CVRS las escalas del } \\
\text { SF-36 que una muestra de población general. }\end{array}$ \\
\hline $\begin{array}{l}\text { Yildirim A. } 2006 . \\
\text { Turquía }{ }^{44} .\end{array}$ & $\begin{array}{c}N: 356 \\
H: 200 \\
M: 156 \\
E M: 43^{\prime} 59\end{array}$ & $\begin{array}{c}\text { PSQ-18 } \\
15 D\end{array}$ & $\begin{array}{l}\text { Estudio } \\
\text { transversal. }\end{array}$ & $\begin{array}{l}\text { CVRS y } \\
\text { satisfacción } \\
\text { de pacientes } \\
\text { trasplantados } \\
\text { renales. }\end{array}$ & $\begin{array}{l}\text { La CVRS fue mejor en receptores de TR masculi- } \\
\text { nos, casados, empleados y con mayor nivel educa- } \\
\text { tivo, frente a pacientes en HD y DP en las mismas } \\
\text { condiciones. }\end{array}$ \\
\hline $\begin{array}{l}\text { Costa-Requena G, } \\
\text { Cantarell Aixendri } \\
\text { MC, Rodriguez Urru- } \\
\text { tia A y Seron Micas } \\
\text { D. 2014.España }{ }^{45} \text {. }\end{array}$ & $\begin{array}{c}N: 72 \\
H: 49 \\
M: 23 \\
E M: 55^{\prime} 75\end{array}$ & $\begin{array}{c}\text { SF-36 } \\
\text { KDQoL-SF }\end{array}$ & $\begin{array}{c}\text { Estudio } \\
\text { descriptivo } \\
\text { longitudinal. }\end{array}$ & $\begin{array}{l}\text { CVRS en } \\
\text { trasplantados } \\
\text { renales a los } \\
6 \text { meses post } \\
\text { trasplante. }\end{array}$ & $\begin{array}{l}\text { Al mes de TR las puntuaciones de CVRS son } \\
\text { inferiores a las de población general. A los } 6 \text { meses } \\
\text { post TR, destacan las dimensiones de rol emocional } \\
\text { y salud mental, mostrándose una CVRS semejante a } \\
\text { la de la población general. }\end{array}$ \\
\hline $\begin{array}{l}\text { Ostrowski M, We- } \\
\text { sołowski T, Makar } \\
\text { D, Bohatyrewicz R. } \\
\text { 2000. Polonia }{ }^{46} \text {. }\end{array}$ & $\begin{array}{c}N: 80 \\
H: 50 \\
M: 30 \\
E M: 18-60\end{array}$ & VAS & $\begin{array}{l}\text { Estudio } \\
\text { observacional } \\
\text { descriptivo. }\end{array}$ & $\begin{array}{l}\text { Cambios en } \\
\text { CVRS tras } \\
\text { el trasplante } \\
\text { renal. }\end{array}$ & $\begin{array}{l}\text { El deterioro de la condición psicofísica en TR es } \\
\text { una consecuencia de hemodiálisis. La mayoría de } \\
\text { los pacientes declaran que el bienestar y CVRS ha } \\
\text { mejorado después de la operación. Tras el injerto } \\
\text { algunos de ellos pueden volver a trabajar y mejorar } \\
\text { su vida social. }\end{array}$ \\
\hline $\begin{array}{l}\text { Ichikawa Y, Fujisawa } \\
\text { M, Hirose E, Kage- } \\
\text { yama T, et al. } 2000 . \\
\text { Japón }{ }^{47} \text {. }\end{array}$ & $\begin{array}{c}\mathrm{N}: 110 \\
\mathrm{H}: 57 \\
\mathrm{M}: 53 \\
\mathrm{EM}: 44^{\prime} 2\end{array}$ & SF-36 & $\begin{array}{c}\text { Estudio } \\
\text { observacional } \\
\text { descriptivo. }\end{array}$ & $\begin{array}{l}\text { CVRS tras } \\
\text { el trasplante } \\
\text { renal. }\end{array}$ & $\begin{array}{l}\text { La creatinina sérica elevada indica peor CVRS y } \\
\text { funcionamiento físico, al igual que las enfermeda- } \\
\text { des comórbidas. Los receptores con episodios de } \\
\text { rechazo expresan peor CVRS en la dimensión rol } \\
\text { emocional. }\end{array}$ \\
\hline
\end{tabular}


[ Carolina Pérez Blancas, et al ]

Factores asociados a la calidad de vida relacionada con la salud de pacientes trasplantados de riñón

\begin{tabular}{|c|c|c|c|c|c|}
\hline $\begin{array}{l}\text { Ponto P, Rupolo G.P, } \\
\text { Marchini F, Feltrin } \\
\text { A, Perin N, Mazzoldi } \\
\text { MA, Giacon B, Baldan } \\
\text { N y Rigotti P. } 2001 . \\
\text { Italia48. }\end{array}$ & $\begin{array}{c}N: 199 \\
H: 131 \\
M: 68 \\
E M: 45^{\prime} 45\end{array}$ & $\begin{array}{c}\text { BSI } \\
\text { LEIPAD } \\
\text { MMSE }\end{array}$ & $\begin{array}{c}\text { Estudio } \\
\text { paralelo: } \\
\text { transversal y } \\
\text { longitudinal. }\end{array}$ & $\begin{array}{l}\text { CVRS tras el } \\
\text { trasplante } \\
\text { renal. Fac- } \\
\text { tores, com- } \\
\text { plicaciones, } \\
\text { cambios. }\end{array}$ & $\begin{array}{l}\text { El género no está asociado como variable de CVRS. } \\
\text { Mayor edad y estar desempleado tienen relación ne- } \\
\text { gativa con CVRS. La CVRS mejora a corto y largo } \\
\text { plazo, pero entre los } 7 \text { y } 36 \text { meses post trasplante se } \\
\text { produce un deterioro de la misma llegando a niveles } \\
\text { pre trasplante. }\end{array}$ \\
\hline $\begin{array}{l}\text { Akman B, } 0 \text { zdemir } \\
\text { FN, Sezer S, Mic, } \\
\text { ozkadiog lu H, and } \\
\text { Haberal M. } 2004 . \\
\text { Turquía }{ }^{49} \text {. }\end{array}$ & $\begin{array}{c}N: 88 \\
H: 62 \\
M: 26 \\
E M: 31^{\prime} 5\end{array}$ & BDI & $\begin{array}{c}\text { Estudio } \\
\text { observacional } \\
\text { descriptivo. }\end{array}$ & $\begin{array}{l}\text { Niveles de } \\
\text { depresión } \\
\text { antes y } \\
\text { después del } \\
\text { trasplante } \\
\text { renal. CVRS. }\end{array}$ & $\begin{array}{l}\text { No se relaciona edad y sexo con nivel de depre- } \\
\text { sión. Estar casado se asocia con menores niveles } \\
\text { de depresión debido a mayor apoyo psicosocial. EI } \\
\text { rechazo agudo del injerto indica mayor porcentaje } \\
\text { depresión. }\end{array}$ \\
\hline $\begin{array}{l}\text { Lazzaretti C.T, } \\
\text { Carvalho J.G.R, } \\
\text { Mulinari R.A, and } \\
\text { Rasia J.M. } 2004 . \\
\text { Brasil }^{50} \text {. }\end{array}$ & $\begin{array}{c}\mathrm{N}: 100 \\
\mathrm{H}: 60 \\
\mathrm{M}: 40 \\
\mathrm{EM}: 36\end{array}$ & WHOQOL- Bref & $\begin{array}{c}\text { Estudio } \\
\text { observacional } \\
\text { descriptivo. }\end{array}$ & $\begin{array}{l}\text { CVRS tras } \\
\text { trasplante } \\
\text { renal. Mejora } \\
\text { multidimen- } \\
\text { sional. }\end{array}$ & $\begin{array}{l}\text { Los pacientes perciben el TR como una mejora de } \\
\text { su CVRS relacionada con la reducción o desapari- } \\
\text { ción de los síntomas causados por la enfermedad } \\
\text { anterior. En los dominios físicos y psicológicos no } \\
\text { hay diferencia significativa. }\end{array}$ \\
\hline $\begin{array}{l}\text { Overbeck I, } \\
\text { Bartels M, Decker } \\
\text { 0, Harms J, Hauss J } \\
\text { y Fangmann J. } 2005 . \\
\text { Alemania }^{51} \text {. }\end{array}$ & $\begin{array}{c}N: 76 \\
H: 49 \\
M: 27 \\
E M: 52^{\prime} 6\end{array}$ & $\begin{array}{c}\text { Giessen-Test } \\
\text { GHQ-28 } \\
\text { SF-36 }\end{array}$ & $\begin{array}{c}\text { Estudio } \\
\text { transversal. }\end{array}$ & $\begin{array}{l}\text { Cambios en la } \\
\text { CVRS tras el } \\
\text { trasplante re- } \\
\text { nal: bienestar } \\
\text { psicológico, } \\
\text { funcional, y } \\
\text { físico. }\end{array}$ & $\begin{array}{l}\text { Los beneficiarios de TR tenían mejores resultados } \\
\text { para el funcionamiento físico, la percepción de salud } \\
\text { general, funcionamiento social y dolor corporal. } \\
\text { Sin embargo no alcanzan los valores medios de } \\
\text { población general. Los pacientes con complicacio- } \\
\text { nes después del injerto mostraron peores niveles } \\
\text { de funcionamiento social, salud mental, rol físico, } \\
\text { mayor nivel de ansiedad e insomnio. El bienestar } \\
\text { psicológico de pacientes fue peor tras } 1 \text { año post } \\
\text { TR que para los trasplantados ese mismo año. Los } \\
\text { pacientes con creatinina sérica elevada presentaron } \\
\text { mayor deterioro de la capacidad cognitiva. }\end{array}$ \\
\hline $\begin{array}{l}\text { Ogutmen B, Yildirim } \\
\text { A, Sever M.S, Boz- } \\
\text { fakioglu S, Ataman } \\
\text { R, Erek E, Cetin 0, } \\
\text { and Emel A. 2006. } \\
\text { Turquía52. }\end{array}$ & $\begin{array}{c}\mathrm{N}: 302 \\
\mathrm{H}: 187 \\
\mathrm{M}: 115 \\
\mathrm{EM}: 38^{\prime} 22\end{array}$ & SF-36 & $\begin{array}{c}\text { Estudio } \\
\text { transversal. }\end{array}$ & $\begin{array}{l}\text { CVRS en } \\
\text { TR, HD y DP. } \\
\text { Factores socio } \\
\text { demográf- } \\
\text { cos, registro } \\
\text { bioquímico. }\end{array}$ & $\begin{array}{l}\text { CVRS se asoció con sexo masculino, }>40 \text { años, estar } \\
\text { casado y empleado. Mejor CVRS en TR que en HD } \\
\text { o DP. Sin embargo, peor que la población general. } \\
\text { Los niveles elevados de hemoglobina y disminui- } \\
\text { dos en creatinina sérica indican mejor CVRS en } \\
\text { pacientes TR. }\end{array}$ \\
\hline $\begin{array}{l}\text { Shah V.S, Ananth A, } \\
\text { Sohal G.K, Bert- } \\
\text { ges-Yost W, Eshelman } \\
\text { A, Parasuraman R.K, } \\
\text { and Venkat K.K. 2006. } \\
\text { Estados Unidos }{ }^{53} \text {. }\end{array}$ & $\begin{array}{l}N N: 50 \\
H: 26 \\
M: 24 \\
E M: 49\end{array}$ & $\begin{array}{l}\text { QLS } \\
\text { BDI y CDI } \\
\text { MSP } \\
\text { IEQ }\end{array}$ & $\begin{array}{c}\text { Estudio } \\
\text { observacional } \\
\text { descriptivo. }\end{array}$ & $\begin{array}{l}\text { CVRS y } \\
\text { factores } \\
\text { psicosociales } \\
\text { asociados a } \\
\text { trasplantados } \\
\text { renales. }\end{array}$ & $\begin{array}{l}\text { La percepción de una mejor CVRS se correlaciona } \\
\text { con menor depresión y enfermedad, mayor apoyo } \\
\text { social y satisfacción. No se observa relación entre } \\
\text { CVRS y edad, tiempo desde el trasplante, creatinina } \\
\text { sérica, hemoglobina o niveles de albúmina. }\end{array}$ \\
\hline $\begin{array}{l}\text { Virzì A, Signorelli } \\
\text { M.S, Veroux M, } \\
\text { Giammarresi G, } \\
\text { Maugeri S, Nicoletti } \\
\text { A, and Veroux P. } 2007 . \\
\text { Italia }{ }^{54} \text {. }\end{array}$ & $\begin{array}{c}\text { N:48 } \\
H: 28 \\
M: 20 \\
\text { EM: } 41^{\prime} 1\end{array}$ & $\begin{array}{l}\text { SF-36 } \\
\text { Hamilton Rating } \\
\text { Scale for } \\
\text { Depression } \\
\text { Hamilton } \\
\text { Anxiety Scale } \\
\text { SAS }\end{array}$ & $\begin{array}{c}\text { Estudio } \\
\text { prospectivo } \\
\text { longitudinal. }\end{array}$ & $\begin{array}{l}\text { CV, depresión } \\
\text { y aspectos } \\
\text { psicológicos } \\
\text { pre- post TR, } \\
\text { en receptores } \\
\text { y donantes. }\end{array}$ & $\begin{array}{l}\text { Mejora de CVRS en todas las dimensiones del } \\
\text { cuestionario SF- } 36 \text { especialmente en las funciones } \\
\text { físicas, actividades sociales, y la salud mental. EI TR } \\
\text { procedente de donante vivo mejora CVRS de los } \\
\text { pacientes. Los niveles de depresión disminuyen post } \\
\text { TR, pero la ansiedad no sufre variación debido al } \\
\text { temor de rechazo del injerto y a los efectos secunda- } \\
\text { rios del tratamiento. }\end{array}$ \\
\hline $\begin{array}{l}\text { Sayin A, Mutluay R, } \\
\text { and Sindel S. } 2007 . \\
\text { Turquía55. }\end{array}$ & $\begin{array}{c}\mathrm{N}: 20 \\
\mathrm{H}: 13 \\
\mathrm{M}: 7 \\
\text { EM: } 33^{\prime} 15\end{array}$ & $\begin{array}{l}\text { SF-36 } \\
\text { BDI } \\
\text { STAI }\end{array}$ & $\begin{array}{l}\text { Estudio } \\
\text { observacional } \\
\text { Análisis de } \\
\text { regresión } \\
\text { lineal } \\
\text { multivariado. }\end{array}$ & $\begin{array}{l}\text { CV,depresión, } \\
\text { ansiedad, } \\
\text { caracterís- } \\
\text { ticas socio } \\
\text { demográficas } \\
\text { y clínicas en } \\
\text { pacientes so- } \\
\text { metidos a HD, } \\
\text { DP Y TR. }\end{array}$ & $\begin{array}{l}\text { Peor CVRS se asoció con sexo masculino, }>46 \text { años, } \\
\text { mayor tiempo en diálisis, presencia de enfermeda- } \\
\text { des comórbidas, donante cadáver. EI menor tiempo } \\
\text { en diálisis disminuye la depresión y ansiedad lo } \\
\text { cual indica mayor CVRS en pacientes TR. Mejores } \\
\text { puntuaciones en TR respecto a HD y DP, pero peor } \\
\text { que población general. }\end{array}$ \\
\hline
\end{tabular}




\begin{tabular}{|c|c|c|c|c|c|}
\hline $\begin{array}{l}\text { Cornella C, Brustia } \\
\text { M, Lazzarich E, } \\
\text { Cofano F, Ceruso A, } \\
\text { Barbé M.C, Fenoglio } \\
\text { R, Cella D and Strat- } \\
\text { ta P. 2008. Italia }{ }^{56} \text {. }\end{array}$ & $\begin{array}{c}N: 52 \\
H: 33 \\
M: 19 \\
E M: 66^{\prime} 8\end{array}$ & SF-36 & $\begin{array}{c}\text { Estudio } \\
\text { observacional } \\
\text { descriptivo. }\end{array}$ & $\begin{array}{l}\text { CV en mayo- } \\
\text { res de } 60 \text { años } \\
\text { trasplantados } \\
\text { renales. Sexo, } \\
\text { dolor, activi- } \\
\text { dad física. }\end{array}$ & $\begin{array}{l}\text { No se observaron diferencias significativas en cuan- } \\
\text { to al género. Los pacientes TR reportan limitaciones } \\
\text { en áreas específicas de la calidad de vida: reducción } \\
\text { de las actividades sociales, mayor percepción del } \\
\text { dolor y el empeoramiento de la salud general. }\end{array}$ \\
\hline $\begin{array}{l}\text { Fujisawa M, Ichikawa } \\
\text { Y, Yoshiya K, Isotani S, } \\
\text { Higuchi A, Nagano S, } \\
\text { Arakawa S, Hamami } \\
\text { G, Matsumoto 0, and } \\
\text { Kamidono S. 2000. } \\
\text { Japón }{ }^{57} \text {. }\end{array}$ & $\begin{array}{c}N: 117 \\
H: 67 \\
M: 50 \\
E M: 33\end{array}$ & SF-36 & $\begin{array}{c}\text { Estudio } \\
\text { observacional } \\
\text { descriptivo. }\end{array}$ & $\begin{array}{l}\text { CV en } \\
\text { pacientes TR } \\
\text { y en lista de } \\
\text { espera some- } \\
\text { tidos a HD. } \\
\text { Edad, fun- } \\
\text { cionamiento } \\
\text { físico y social, } \\
\text { dolor, nivel de } \\
\text { creatinina. }\end{array}$ & $\begin{array}{l}\text { Los pacientes con TR tuvieron puntuaciones signifi- } \\
\text { cativamente más altas en el funcionamiento físico, } \\
\text { dolor corporal, salud general, y escala de funciona- } \\
\text { miento social respecto a los pacientes en HD. Los } \\
\text { niveles más bajos de creatinina sérica y edad menor } \\
\text { de } 40 \text { años se asociaron con mayor CVRS (mayor } \\
\text { funcionamiento físico, salud general y vitalidad). }\end{array}$ \\
\hline $\begin{array}{l}\text { Karam V.H, Gasquet } \\
\text { I, Delvart V, Hiesse } \\
\text { C, Dorent R, Danet C, } \\
\text { Didier S, Charpentier } \\
\text { B, Gandjbakhch I, } \\
\text { Bismuto H, y Castaing } \\
\text { D. 2003. Francia } \\
\text {. }\end{array}$ & $\begin{array}{c}\mathrm{N}: 229 \\
\mathrm{H}: 121 \\
\mathrm{M}: 108 \\
\mathrm{EM}: 52^{\prime} 8\end{array}$ & NIDDKQOL & $\begin{array}{c}\text { Estudio } \\
\text { multicéntrico } \\
\text { transversal. }\end{array}$ & $\begin{array}{l}\text { CV trasplante } \\
\text { de riñón, híga- } \\
\text { do y corazón } \\
\text { tras } 10 \text { años. }\end{array}$ & $\begin{array}{l}\text { Los pacientes TR presentan peor CVRS que la } \\
\text { población general, salvo en el área de depresión y } \\
\text { angustia donde no existe diferencia entre ambos. } \\
\text { Los receptores de TR presentan peor estado psico- } \\
\text { lógico que otros trasplantes de órganos sólidos, así } \\
\text { como peor CVRS a largo plazo, >10 años. }\end{array}$ \\
\hline $\begin{array}{l}\text { Hongxia Liu, Irene } \\
\text { Feurer, Kathleen Dw- } \\
\text { yer, David Shaffer \& C. } \\
\text { Wright Pinson.2019. } \\
\text { EEUU }{ }^{12} \text {. }\end{array}$ & $\begin{array}{c}\mathrm{N}: 16 \\
\mathrm{H}: 86 \\
M: 74 \\
E M: 47^{\prime} 7\end{array}$ & $\begin{array}{l}\text { SF-36 } \\
\text { CAHS } \\
\text { Brief COPE } \\
\text { PRQ2000 } \\
\text { PHCS }\end{array}$ & $\begin{array}{l}\text { Diseño } \\
\text { descriptivo } \\
\text { transversal. }\end{array}$ & $\begin{array}{l}\text { CVRS, facto- } \\
\text { res clínicos, } \\
\text { psicosociales, } \\
\text { superación, } \\
\text { autoeficacia } \\
\text { en trasplanta- } \\
\text { dos renales. }\end{array}$ & $\begin{array}{l}\text { El tratamiento inmunosupresor provoca efectos } \\
\text { secundarios en los pacientes con TR que influyen } \\
\text { de manera negativa sobre su CVRS. Los pacientes } \\
\text { con menos de } 1 \text { año después del TR utilizan más el } \\
\text { compromiso de afrontamiento, tienen mayor grado } \\
\text { de autoeficacia percibida y apoyo social que los que } \\
\text { llevan más tiempo trasplantados. En general, pre- } \\
\text { sentan CVRS física menor que la población general } \\
\text { y CVRS mental similar. }\end{array}$ \\
\hline $\begin{array}{l}\text { Matas AJ, Halbert RJ, } \\
\text { Barr ML, Helderman } \\
\text { JH, Hricik DE, Pirsch } \\
\text { JD, Schenkel FA, } \\
\text { Siegal BR, Liu H, } \\
\text { Ferguson RM. 2002. } \\
\text { EEUU }{ }^{59} \text {. }\end{array}$ & $\begin{array}{c}N: 4247 \\
H: 2022 \\
M: 2225 \\
E M: 51\end{array}$ & $\begin{array}{l}\text { TCI } \\
\text { LSI }\end{array}$ & $\begin{array}{l}\text { Estudio } \\
\text { observacional } \\
\text { Análisis de } \\
\text { regresión } \\
\text { múltiple. }\end{array}$ & $\begin{array}{l}\text { Satisfacción } \\
\text { y efectos } \\
\text { adversos en } \\
\text { trasplantados } \\
\text { renales. }\end{array}$ & $\begin{array}{l}\text { La mejora de CVRS se asocia con sexo masculino, } \\
\text { edad avanzada, menor número de comorbilidades, } \\
\text { vivir en pareja y menor disfunción sexual. La gran } \\
\text { mayoría de los pacientes TR refieren efectos adver- } \\
\text { sos como consecuencia del tratamiento inmunosu- } \\
\text { presor. }\end{array}$ \\
\hline
\end{tabular}

N: muestra, H:hombres, M: mujeres, EM:edad media, SF- 36;MOS SF 36: Cuestionario de evaluación de CVRS, BDI: Inventario de evaluación de la depresión, ReTransQol: instrumento de medida de CVRS, Escala Karnofsky: escala de evaluación de CVRS, WHOQOL-Bref :instrumento de evaluación de la CVRS según la OMS, MLDL: Cuestionario de medida de las dimensiones de CVRS, BSI: inventario breve para medir síntomas, WHOQOL 100: cuestionario de medida de CVRS, EuroQol 5-D: cuestionario descriptivo del estado de salud, ESRD-SCL: cuestionario para evaluar CVRS en receptores de trasplante renal teniendo en cuenta los efectos de la terapia inmunosupresora, SF-12: cuestionario de salud, GBB-24: cuestionario de medición de quejas físicas subjetivas, Bf-S: escala de medición del estado de ánimo, PSQ-18 y 15D: cuestionario de evaluación de la satisfacción y CVRS, KDQoL-SF: Cuestionario de calidad de vida de enfermedades renales, VAS: escala visual analógica, LEIPAD: cuestionario específico para evaluar la calidad de vida y el registro de los principales detalles personales y profesionales, MMSE: mini examen mental para medir el deterioro cognitivo, Giessen-Test: prueba de personalidad, GHQ-28: instrumento de medición de la ansiedad y depresión, QLS: escala de calidad de vida, CDI: escala de medición del índice cognitivo de depresión, IEQ: cuestionario de medición de los efectos de la enfermedad, MSP: escala multidimensional de percepción social, SAS: cuestionario de auto calificación de ansiedad, STAI: inventario de evaluación del estado y rango de ansiedad, NIDDKQOL: cuestionario de evaluación de la calidad de vida de los pacientes trasplantados, CASH: escala de evaluación de la salud cognitiva, PHCS: escala de percepción de salud, PRQ2000: escala de recursos personales para medir la percepción de apoyo social, Brief COPE: escala abreviada de medición del nivel de afrontamiento, TCI: instrumento de medida de los problemas relacionados con el cuidado del trasplante, LSI: escala de evaluación de la satisfacción y calidad de vida. 
En la tabla III están representados los estudios que comparan la CVRS entre las diferentes modalidades de TRS y la población sana.

Tabla III. Comparación CVRS en TR, HD, DP y población sana

\begin{tabular}{|l|l|l|l|l|}
\hline $\begin{array}{l}\text { Dimensiones } \\
\text { SF-36 (0-100) puntos) }\end{array}$ & $\begin{array}{l}\text { Trasplante renal } \\
16,27,28,3342,52,55\end{array}$ & $\begin{array}{l}\text { Hemodiálisis } \\
16,27,28,42,52,55\end{array}$ & $\begin{array}{l}\text { Diálisis Peritoneal } \\
27,42,52,55\end{array}$ & $\begin{array}{l}\text { Población sana } \\
16,33,42,52\end{array}$ \\
\hline Funcionamiento físico (FF) & $59^{\prime} 92-85^{\prime} 8$ & $47^{\prime} 5-56^{\prime} 99$ & $52^{\prime} 14-57^{\prime} 06$ & $61^{\prime} 80-92^{\prime} 2$ \\
\hline Rol físico (RF) & $42^{\prime} 50-81^{\prime} 3$ & $10^{\prime} 2-64^{\prime} 4$ & $35^{\prime} 7-55^{\prime} 10$ & $76^{\prime} 2-83^{\prime} 6$ \\
\hline Funcionamiento social (FS) & $57^{\prime} 93-93^{\prime} 2$ & $30^{\prime} 9-78^{\prime} 9$ & $56^{\prime} 32-74^{\prime} 11$ & $70^{\prime} 3-90^{\prime} 1$ \\
\hline Rol emocional (RE) & $45^{\prime} 6^{\prime}-93^{\prime} 1$ & $39^{\prime} 6-72^{\prime} 7$ & $46^{\prime} 14-73^{\prime} 81$ & $74^{\prime} 44-88^{\prime} 6$ \\
\hline Dolor corporal (DC) & $64^{\prime} 00-90^{\prime} 2$ & $45^{\prime} 50-70^{\prime} 02$ & $54^{\prime} 6-74^{\prime} 34$ & $70^{\prime} 02-84^{\prime} 8$ \\
\hline Vitalidad (V) & $55^{\prime} 71-83^{\prime} 00$ & $25^{\prime} 9-56^{\prime} 20$ & $31^{\prime} 9-55^{\prime} 51$ & $58^{\prime} 79-68^{\prime} 2$ \\
\hline Salud general (SG) & $43^{\prime} 81-84^{\prime} 00$ & $28^{\prime} 00-50^{\prime} 08$ & $41^{\prime} 25-50^{\prime} 66$ & $45^{\prime} 8-68^{\prime} 3$ \\
\hline Salud mental (SM) & $63^{\prime} 43-83^{\prime} 7$ & $23^{\prime} 4-67^{\prime} 1$ & $47^{\prime} 5-68^{\prime} 29$ & $63^{\prime} 7-73^{\prime} 00$ \\
\hline
\end{tabular}

A continuación están representados los valores medios de CVRS en las ocho dimensiones del SF-36, comparando los pacientes con TR, con HD y DP y población sana (Fig. 2).

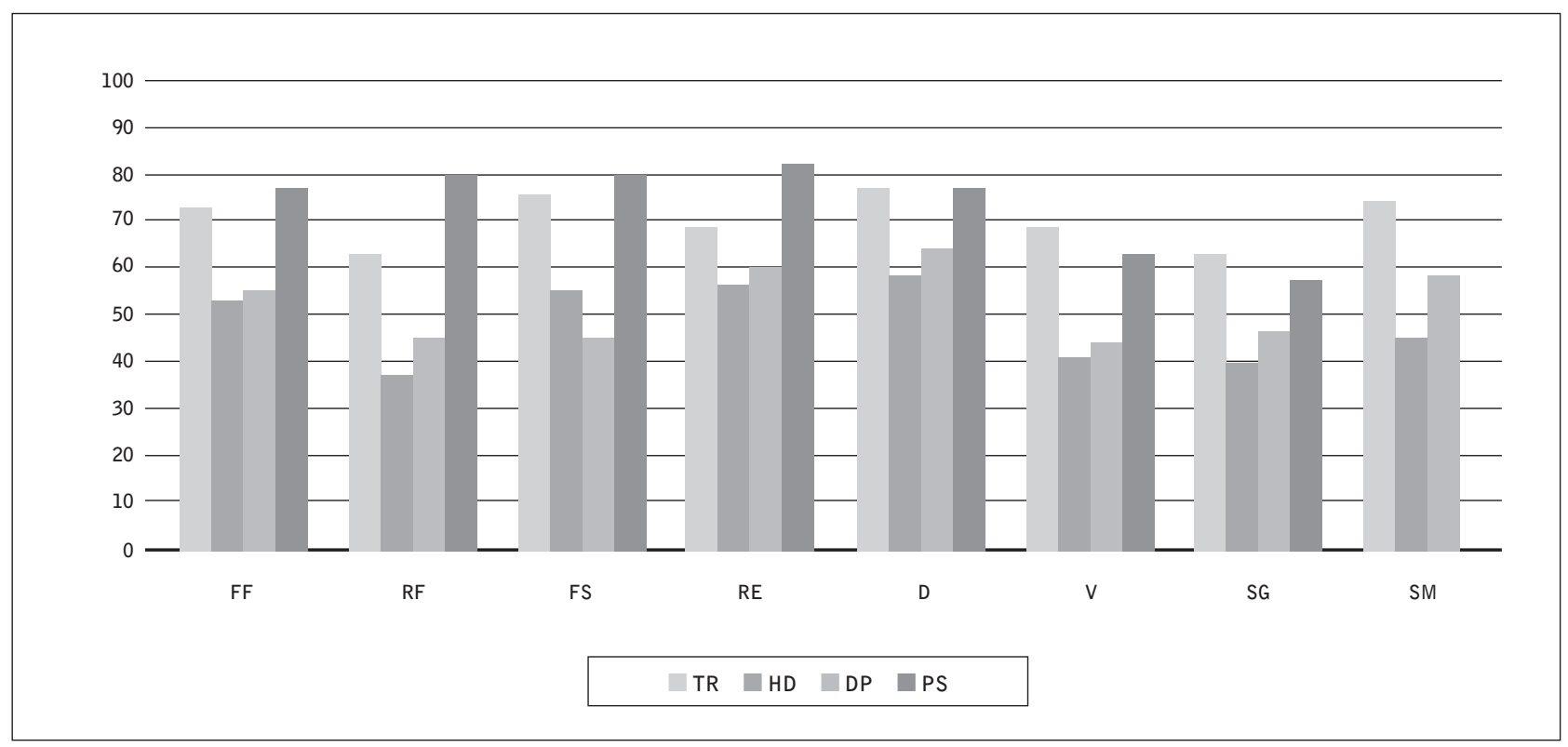

Figura 2. Representación gráfica de CVRS entre TR, HD, DPY PS.

Finalmente en la tabla IV se han recogido los estudios que comparaban la CVRS antes y después del TR, teniendo en cuenta que no está determinado el tiempo entre la determinación Pre-TR y la realización, y sobre todo, la determinación Post-TR está tomada a tiempos diferentes en cada estudio. 
Tabla IV. Comparación CVRS en TR, HD, DP y población sana

\begin{tabular}{|c|c|c|c|c|}
\hline \multirow{2}{*}{$\begin{array}{c}\text { Revisión } \\
\text { bibliográfica }\end{array}$} & \multirow{2}{*}{ Muestra } & \multirow{2}{*}{$\begin{array}{c}\text { Instrumento } \\
\text { y dimensión estudiada }\end{array}$} & \multicolumn{2}{|c|}{ Resultados } \\
\hline & & & Pre TR & Post TR \\
\hline $\begin{array}{l}\text { Oliveira de Mendonça AE , De } \\
\text { Góes Salvetti M, Chaves Maia } \\
\text { EM , De Oliveira e Silva AC, } \\
\text { De Vasconcelos Torres G. } 2015 . \\
\text { Brasil }^{34} \text {. }\end{array}$ & $\begin{array}{c}N: 63 \\
H: 39 \\
M: 24 \\
E M: 39^{\prime} 9\end{array}$ & $\begin{array}{l}\text { WHOQOL-Bref. } \\
\text { Dominio físico. }\end{array}$ & $\begin{array}{l}\text { Puntuación media 9’94. } \\
\text { Correlación positiva-débil } \\
\text { entre CVRS, energía, sueño } \\
\text { y capacidad para las ABVD } \\
\text { (puntuaciones bajas). } \\
\text { Correlación negativa-débil } \\
\text { entre CVRS y necesidad de } \\
\text { tratamiento (mayor necesidad } \\
\text { de tratamiento). } \\
\text { Correlación negativa-modera- } \\
\text { da entre CVRS y el impacto } \\
\text { del dolor en actividades } \\
\text { (puntuaciones altas de dolor } \\
\text { y fatiga). }\end{array}$ & $\begin{array}{l}\text { Puntuación media } 17^{\prime} 41 . \\
\text { Correlación positiva-débil en- } \\
\text { tre CVRS y energía, capacidad } \\
\text { de moverse y trabajar. } \\
\text { Correlación negativa modera- } \\
\text { da entre CVRS y necesidad de } \\
\text { tratamiento. } \\
\text { Mejoran todas las facetas } \\
\text { físicas mostrando percepción } \\
\text { positiva de CVRS. Menor } \\
\text { necesidad de tratamiento y } \\
\text { reducción al mínimo el impac- } \\
\text { to del dolor son los datos más } \\
\text { relevantes. }\end{array}$ \\
\hline $\begin{array}{l}\text { Ortega T, Deulofeu R, Salamero } \\
\text { P, Roman A, Masnou N, Rubio } \\
\text { N, Garcia 0, Casanovas T, } \\
\text { Cofán F, Twose J, Ortega F. } \\
\text { 2009. España }{ }^{37} \text {. }\end{array}$ & $\begin{array}{l}N: 126 \\
H: 85 \\
M: 41 \\
E M: 53\end{array}$ & $\begin{array}{l}\text { SF-36. } \\
\text { Componente físico y } \\
\text { componente mental. }\end{array}$ & $\begin{array}{l}\text { Puntuaciones bajas en todas } \\
\text { las subescalas de componente } \\
\text { físico y componente mental. } \\
\text { Los valores más bajos se } \\
\text { encuentran en la subescala } \\
\text { funcionamiento social. }\end{array}$ & $\begin{array}{l}12 \text { meses post TR: puntua- } \\
\text { ciones más altas en todas } \\
\text { las subescalas del SF- } 36 \text { lo } \\
\text { que implica una mejora de la } \\
\text { CVRS tanto en componente } \\
\text { físico como mental, aunque } \\
\text { prevalece la mejora de CVRS } \\
\text { sobre el componente mental. } \\
\text { Los valores medios de dolor } \\
\text { corporal son mayores en esta } \\
\text { fase en <50 años y en muje- } \\
\text { res mayores de } 50 \text { años. }\end{array}$ \\
\hline $\begin{array}{l}\text { Rodríguez Martín MC, Fernán- } \\
\text { dez Zapico D, Fuenmayor Díaz } \\
\text { A, et al. 2009. España }{ }^{18} \text {. }\end{array}$ & $\begin{array}{c}\mathrm{N}: 31 \\
\mathrm{H}: 14 \\
\mathrm{M}: 17 \\
\mathrm{EM}: 71^{\prime} 8\end{array}$ & SF-36. & $\begin{array}{l}\text { Media }(0-10) \text { de percepción } \\
\text { de CVRS de } 2^{\prime} 94 \text {. Grandes } \\
\text { limitaciones a la hora de } \\
\text { realizar esfuerzos físicos. }\end{array}$ & $\begin{array}{l}\text { 2'77 años post TR: media } \\
\text { de 9'13. La función física } \\
\text { sigue siendo el aspecto peor } \\
\text { valorado. } \\
\text { - } 36 \% \text { Mejor CVRS } \\
\text { - } 42 \% \text { Igual } \\
\text { - } 6 \% \text { Peor }\end{array}$ \\
\hline $\begin{array}{l}\text { Ariana Paola Canché-Arenas, } \\
\text { Marco Reza-Orozco, Federico } \\
\text { Leopoldo Rodríguez-We- } \\
\text { ber.2011. México }{ }^{38} \text {. }\end{array}$ & $\begin{array}{l}N: 30 \\
H: 19 \\
M: 11 \\
E M: 49\end{array}$ & Escala Karnofsky. & $\begin{array}{l}\text { Calidad de vida menor al } 60 \% \\
\text { (requieren atención ocasional, } \\
\text { asistencia y cuidados médicos } \\
\text { frecuentes, hospitalización } \\
\text { indicada). }\end{array}$ & $\begin{array}{l}\text { Calidad de vida superior al } \\
70 \% \text {. Pasaron a ser inde- } \\
\text { pendientes sólo con algunos } \\
\text { cuidados para su enfermedad. } \\
\text { Leves dificultades para reali- } \\
\text { zar actividades o trabajo acti- } \\
\text { vo. Aumento de } 20-60 \text { puntos } \\
\text { en todos los casos el valor del } \\
\text { karnofsky. Mejor CVRS al no } \\
\text { depender de diálisis ni de ter- } \\
\text { ceros y presentar disminución } \\
\text { de los síntomas y restricciones } \\
\text { del movimiento. }\end{array}$ \\
\hline
\end{tabular}




\begin{tabular}{|c|c|c|c|c|}
\hline $\begin{array}{l}\text { Pérez San Gregorio M.A, } \\
\text { Martín Rodríguez A, Díaz } \\
\text { Domínguez R, y Pérez Bernal } \\
\text { J. 2007. España }{ }^{24} \text {. }\end{array}$ & $\begin{array}{c}\mathrm{N}: 28 \\
\mathrm{H}: 15 \\
\mathrm{M}: 13 \\
\text { EM: } 40^{\prime} 61\end{array}$ & $\begin{array}{c}\text { SF-36. } \\
\text { EuroQol. } \\
\text { ESRD-SCL. }\end{array}$ & $\begin{array}{l}\text { Puntuaciones medias bajas en } \\
\text { todas las dimensiones de los } \\
\text { tres cuestionarios analizados } \\
\text { comparado con la etapa post } \\
\text { TR. }\end{array}$ & $\begin{array}{l}\text { 3, } 6, \text { y } 12 \text { meses post-TR: } \\
\text { resultados significativos en las } \\
\text { dimensiones funcionamiento } \\
\text { físico, limitaciones en rol de } \\
\text { problemas físicos, vitalidad, } \\
\text { salud mental, actividades } \\
\text { cotidianas, salud actual, } \\
\text { disfunción cardiaca y renal } \\
\text { y efectos secundarios de los } \\
\text { esteroides. Excepto en esta } \\
\text { última, en la que se aprecia } \\
\text { un empeoramiento a medida } \\
\text { que transcurre el tiempo, en } \\
\text { el resto se observan mejorías } \\
\text { post TR. En la fase pre-TR y } \\
6 \text { meses post-TR hay mejoría } \\
\text { en vitalidad y salud mental. La } \\
\text { salud actual también mejora } \\
\text { entre la fase pre, } 3 \text { y } 6 \text { meses } \\
\text { post, así como la disfunción } \\
\text { cardiaca que mejora a largo } \\
\text { plazo. }\end{array}$ \\
\hline $\begin{array}{l}\text { Virzì A, Signorelli M.S, Veroux } \\
\text { M, Giammarresi G, Maugeri S, } \\
\text { Nicoletti A, and Veroux P. } 2007 . \\
\text { Italia }^{54} \text {. }\end{array}$ & $\begin{array}{c}\mathrm{N}: 48 \\
\mathrm{H}: 28 \\
\mathrm{M}: 20 \\
\text { EM: } 41^{\prime} 1\end{array}$ & $\begin{array}{c}\text { SF-36. } \\
\text { Hamilton Rating } \\
\text { Scale for Depression, } \\
\text { Hamilton Anxiety Scale } \\
\text { SAS. }\end{array}$ & $\begin{array}{l}\text { Presencia de síntomas depre- } \\
\text { sivos asociados a la IRCT y } \\
\text { a la angustia psicológica que } \\
\text { provoca el periodo en diálisis. }\end{array}$ & $\begin{array}{l}\text { Mejora de la CVRS en rol fí- } \\
\text { sico (limitación en actividades } \\
\text { físicas), actividades sociales y } \\
\text { salud mental. } \\
\text { No existe variación en los } \\
\text { niveles de ansiedad debido } \\
\text { al temor a rechazo y a los } \\
\text { efectos secundarios del trata- } \\
\text { miento inmunosupresor, pero } \\
\text { sí se observa una disminución } \\
\text { significativa en los nivel de } \\
\text { depresión. }\end{array}$ \\
\hline
\end{tabular}

\section{Descripción de los resultados}

\section{Variables asociadas a CVRS}

\section{Género}

Varios estudios exponen que no existen diferencias en cuanto a género y consideran que no es una variable a tener en cuenta a la hora de medir la CVRS $23,26,28,35,48,56,57$. En cambio, otros estudios muestran controversias respecto a la mejora de CVRS asociada al género. Por un lado hay autores que afirman que es el sexo masculino el que presenta asociada mayor CVRS frente al sexo femenino $29,30,31,44,52,59$. Por otro lado, aunque en menor proporción, las mujeres cuentan con mejores estrategias de afrontamiento, mayor adaptación a la enfermedad y puntuaciones superiores en dimensiones de salud psicológica, relaciones sociales, espiritualidad y calidad de vida global que los hombres $33,3,14,55$.

\section{Edad}

La edad avanzada está asociada con mayor CVRS $3,37,39,40,45,52,59$ y una disminución de los efectos secundarios de tratamientos inmunosupresores. Hay estudios que documentan que las personas con edad avanzada presentan menos preocupaciones y angustia por perder el injerto en comparación con las personas jóvenes, debido a una mayor estabilidad psicológica ${ }^{30,35}$. Otro estudio ha encontrado puntuaciones más altas en mayores de 65 años en solución de problemas, confrontación, reevaluación positiva, salud psicológica, nivel de independencia, ambiente y espiritualidad3. Los receptores de trasplante renal que pertenecen a la categoría de adultos jóvenes, se han calificado con bajas puntuaciones en las dos escalas del componente físico y mental, presentando mayores diferencias en salud general, limitaciones de rol debido a problemas de conducta y físicos ${ }^{43}$. 
Sin embargo, otros estudios encontraron una relación entre más edad ( $>$ de 65 años) con peor CVRS $^{14,22,2}$ $3,27,30,31,32,36,48,53$, sobre todo de componente físico, con puntuaciones más bajas comparadas con personas más jóvenes en las dimensiones de: funcionamiento físico $\left(71^{\prime} 2\right.$ frente a $\left.84^{\prime} 5\right)$, funcionamiento social $\left(67^{\prime} 5\right.$ frente a $\left.84^{\prime} 5\right)$, rol emocional $\left(50^{\prime} 6 \text { frente a } 78^{\prime} 8\right)^{36}$, presentando mas limitaciones en la actividad física que los pacientes más jóvenes ${ }^{18,28,29}$. No obstante, varios estudios no han encontrado diferencias en la CVRS entre distintos grupo de edad ${ }^{16,45}$.

\section{Estado civil}

El estado civil como variable asociada a la CVRS no parece influir de manera significativa, pero pese a ello, se han encontrado estudios en los que se relaciona una mejora de la CVRS con pacientes que se encuentran casados o con pareja y ser soltero, viudo o vivir solo se asocia con peor CVRS debido a un menor apoyo familiar3,30,36,44,49,52,59.

\section{Nivel de estudios}

Se ha encontrado una correlación entre CVRS y satisfacción, y tener estudios. A mayor nivel de estudios, mayor CVRS percibida por los pacientes. Los pacientes con estudios universitarios tienen una media superior al resto de paciente en comparación con el resto $\left(47^{\prime} 89\right.$ vs $\left.43^{\prime} 68\right)$, asociándose además, con una media más alta en las dimensiones de salud psicológica y relaciones sociales ${ }^{21,22,30,31,44}$.

\section{Datos bioquímicos}

Algunas series no han encontrado relación entre niveles séricos de hemoglobina y albúmina y CVRS 3,35 . En cambio, otros estudios comunican que un aumento de creatinina sérica y una disminución del hematocrito disminuyen la puntuación en el resumen del componente físico ( $11 \%$ ) y también tiene efecto negativo sobre la salud general y el funcionamiento físico, y la vitalidad $29,33,57$. Otros autores han encontrado que niveles altos de hemoglobina favorece una buena calidad de vida, disminuye la mortalidad y mejora la tolerancia al ejercicio ya que actúa sobre la función miocárdica ${ }^{31,32}$.

En otro grupo de pacientes se ha encontrado que con cifras de hemoglobina $11.6-13.13 \mathrm{mg} / \mathrm{dl}$, de albúmi- na sérica superior a $34 \mathrm{mg} / \mathrm{dl}$ refieren mejor CVRS en pacientes trasplantados en comparación con HD, pero peor que la población general de su misma edad y $\operatorname{sex} 0^{14,32,52}$.

\section{Empleo}

Algunos estudios recogen que los pacientes con TR que tienen empleo, expresan mayor CVRS y satisfacción $48^{\prime} 46$ frente al resto $43^{\prime} 89^{23,44}$. En una serie se comparan a los pacientes desempleados con los empleados, encontrándose que estos últimos tenían mejores puntuaciones medias en funcionamiento físico ( $83^{\prime} 7$ vs $\left.73^{\prime} 8\right)$, rol físico $\left(67^{\prime} 9\right.$ vs $\left.83^{\prime} 6\right)$, salud general (63'6 vs 51'6), rol emocional ( $78^{\prime} 3$ vs $54^{\prime} 8$ ) (35). Otros estudios, recogen que entre $25-72 \%$ de desempleados refieren mala CVRS y entre $46-74 \%$ estaban satisfechos con su trabajo y ocupación ${ }^{43,48,50}$.

Una publicación muestra los porcentajes respecto al empleo antes y después del TR, lo cual se traduce en una reducción del $72 \%$ del número de pacientes desempleados, tabla $\mathbf{V}^{46}$.
Tabla V. Porcentaje de empleados antes y después del TR

\begin{tabular}{|l|l|}
\hline Antes del trasplante: & Después del trasplante: \\
\hline$\cdot 622^{\prime} 5 \%$ desempleados & $\cdot 18^{\prime} 5 \%$ se mantuvieron desempleados \\
$\cdot \cdot 15 \%$ empleo a tiempo parcial & $\cdot 63^{\prime} 5 \%$ empleo a tiempo parcial \\
$\cdot \cdot 22^{\prime} 5 \%$ empleo a tiempo completo & $\cdot 10 \%$ empleo a tiempo completo
\end{tabular}

El hecho de estar desempleado indica la existencia de valores más bajos en el componente mental29,30.

\section{Ingresos económicos}

Mayores ingresos económicos y pacientes con renta más alta se correlaciona con mejor percepción de su estado físico y mayor CVRS mental ${ }^{22,29}$. La mayoría de los receptores no observan diferencias en cuanto a los ingresos económicos tras el injerto $89^{\prime} 2 \%, 9$, $2 \%$ tuvieron ingresos superiores tras la intervención y 1'5\% los vieron disminuidos, según un artículo35. Sin embargo, otro artículo muestra peor CVRS relacionado con ingresos medios-altos ${ }^{55}$.

\section{Tratamiento inmunosupresor}

El uso de medicación inmunosupresora se ha asociado con una disminución de la CVRS27. El 79\% de los receptores de TR expresan efectos secundarios 
del tratamiento inmunosupresor relacionados con la modificación del cuerpo ( $72^{\prime} 7 \%$ ) (Aumento de peso, cansancio, insomnio, estrés, diabetes, hipercolesterolemia, HTA), lo que conlleva menor cumplimiento de la adherencia terapéutica ${ }^{12,30,46,59}$.

Otro estudio encuentra que el $54^{\prime} 6 \%$ de los receptores tratados con tratamiento inmunosupresor no presentan efectos secundarios asociados, $28^{\prime} 5 \%$ tienen problemas dermatológicos, $28 \%$ refieren problemas músculo-esqueléticos, y el $25^{\prime} 5 \%$ problemas gastrointestinales ${ }^{23}$. .Aún así, el 85\% tiene buena adherencia al tratamiento ${ }^{55}$. Tan sólo un estudio ha expresado que el tratamiento inmunosupresor no es indicativo de CVRS ${ }^{31}$.

\section{Problemas psicológicos}

Las variables psicológicas se asocian a la CVRS en el componente físico. En los grupos más jóvenes se asocia con neuroticismo $\left(40^{\prime} 7 \%\right)$, en el grupo de $40-55$ años se asocia con neuroticismo (20'8\%) y trastorno psicológico (19'2\%), y en el grupo de mayores de 55 años sólo se asocia con trastorno psicológico $27 \% 7 \%$. La angustia psicológica tiene correlación negativa con función física $\left(5^{\prime} 5 \%\right)$, limitación de rol físico (3'5\%), dolor corporal (13'3\%), y percepción de salud general $\left(6^{\prime} 7 \%\right)$. Sin embargo, el neuroticismo solo tiene correlación negativa con la función física (4'4\%) y el dolor corporal (4'8\%). De manera que cuanto menor sea el neuroticismo y la angustia psicológica mayor CVRS ${ }^{22}$.

La depresión es otro problema psicológico que puede aparecer en pacientes con TR. Se han encontrado datos acerca de que las cifras de depresión son similares en la población general, $18 \% 26,58$. Según un estudio las mujeres y jóvenes son más propensas a sufrir depresión con una frecuencia del $25-30 \%$, así como una corta duración del buen funcionamiento del injerto a causa del rechazo agudo del mismo, lo que implicaría el retorno a HD. Aquellos pacientes que se encuentran casados también expresan menores niveles de depresión debido al apoyo psicosocial. Pese a estos datos, los valores medios de depresión son menores tras el TR que durante la estancia en HD, debido a que el trasplante conduce a la reintegración al disminuir la depresión y aumentar la motivación e integración social ${ }^{51,53}$. Sin embargo, otro estudio indica que la depresión está vinculada a HD y DP pero no al $\mathrm{TR}^{55}$. Los síntomas que aparecen como consecuencia de los efectos secundarios de los tratamientos inmunosupresores, dan lugar a la necesidad de un mayor afrontamiento re- lacionado con menor grado de autoeficacia percibida, influyendo a su vez en menor CVRS física y mental ${ }^{10}$. En comparación con otros trasplantes de diferentes órganos sólidos el TR presenta peor estado psicológico que el resto ${ }^{58}$.

\section{Diagnóstico principal de enfermedad}

Los principales diagnósticos de IRCT encontrados son: ${ }^{26,28}$.

- Glomerulonefritis 31\% - 38\%

- Nefropatía diabética $17 \%$

- Nefropatía hipertensiva 38\%

- Pielonefritis crónica 22 '3\%

- Causa desconocida $8 \%$

Hay autores que entre sus datos recogidos encuentran una diferencia de diagnóstico principal de la IRCT en función de la edad; señalando la diabetes ( $81 \%$ ) en los pacientes trasplantados renales más jóvenes, y la glomerulonefritis $(26 \%)$ entre los receptores mayores de 65 años ${ }^{16}$.

\section{Tiempo trasplante}

Más tiempo post trasplante indica mejor CVRS mental debido a una mejor adaptación y buen funcionamiento del injerto 22,26 . Sin embargo, hay estudios que recogen esta variable con una correlación negativa con la CVRS y el componente mental, asociando menos tiempo trasplantado con mayor CVRS (puntuación media $=48^{\prime} 74$ ), más tiempo trasplantado con menor CVRS puesto que aumentan los síntomas físicos, la incertidumbre, fatiga y miedo (puntuación me$\left.\mathrm{dia}=46^{\prime} 06\right)^{23,39}$.

La CVRS física disminuye desde el inicio hasta los 6 años post-trasplante. A diferencia de los indicadores de CVRS mental que mejoran, aumentando con el tiempo: salud mental, vitalidad. La única excepción fue la limitación de rol por problemas emocionales que obtuvo peor puntuación, como puede apreciarse en la tabla VI ${ }^{35}$.

Tabla VI. Cambios en el resumen de componente físico y mental

\begin{tabular}{l|l|}
\hline $\begin{array}{l}\text { Cambios con respecto } \\
\text { al componente físico } \\
\text { (6 años post- trasplante): }\end{array}$ & $\begin{array}{l}\text { Cambios con respecto al } \\
\text { componente mental (6 años } \\
\text { post- trasplante) : }\end{array}$ \\
\hline$\cdot 30^{\prime} 7 \%$ no presento cambios. & $\cdot 45^{\prime} 3 \%$ no presentó cambio \\
\hline$\cdot 53^{\prime 3} \%$ agravó & $\cdot 33^{\prime 3} \%$ agravó \\
\hline$\cdot 16 \%$ mejoró & $\cdot 21,3 \%$ mejoró \\
\hline
\end{tabular}


Sin embargo, según un estudio italiano, en el periodo de 7-36 meses post trasplante la CVRS vuelve a los niveles de deterioro de la etapa pre trasplante ${ }^{48}$. Otros autores refieren que a medio plazo (5-10 años) después del TR se obtienen las mayores puntuaciones del SF-36, mientras que después de los 10 años disminuyen debido a mayor edad y a los efectos secundarios de los inmunosupresores 47,58 . A largo plazo los receptores de TR presentan puntuaciones más bajas en todas las escalas que la población general:

- Funcionamiento físico: $80^{\prime 2} 2$ vs $92^{\prime 2} 2$.

- Rol físico: $67^{\prime} 9$ vs $83^{\prime} 6$.

- Salud general: 59'4 vs 69'2.

- Vitalidad: 61'4 vs 68'2.

- Rol emocional: 70 vs $79^{\prime} 4$.

- Salud mental: 68'4 vs 73 .

- Dolor corporal: 82'5 vs 84'5. (Esta dimensión es la más cercana a la población general).

\section{Diálisis pre-trasplante}

El tiempo en diálisis previo al TR, se correlaciona negativamente con calidad de vida, es decir a mayor tiempo en diálisis disminuye la supervivencia de 5 a 10 años y la CVRS $^{30,31,55}$. Los receptores que estuvieron más de 3 años en diálisis expresan peor CVRS tras el trasplante que aquellos que estuvieron un periodo más corto ${ }^{51}$. Además un menor tiempo en diálisis está vinculado a una disminución de la depresión y la ansiedad ${ }^{55}$. El componente físico también tiene una relación directa con el tiempo en diálisis de manera que mayor tiempo en este tipo de TRS implica peor CVRS ${ }^{39}$.

\section{Trasplante vivo vs trasplante cadáver}

Receptores de injertos de cadáver refieren peor nivel en los componentes dolor corporal, salud general, y funcionamiento físico en comparación con los pacientes con injertos procedente de donante vivo ${ }^{28,55}$. EITR de donante vivo aumenta el funcionamiento físico desde el inicio del trasplante en comparación con el injerto de cadáver, pero provoca mayor deterioro $0^{35}$. El resto de datos encontrados en los diversos estudios no son muy significativos ya que no tienen gran evidencia. Hay autores que indican peor CVRS en injerto de vivo y para otros en cambio que no existe diferencia significativa entre donante vivo 0 cadáver ${ }^{57}$.

\section{Comorbilidad asociada}

Cuando se asocian comorbilidad y efectos secundarios del tratamiento en el TR, acarrean un deterioro del com- ponente físico importante $28,31,55,59$. La hipertensión arterial $65^{\prime} 4 \%$, diabetes $54 \%$, anomalías óseas $7^{\prime} 6 \%$ así como otras comorbilidades y disfunción inicial del injerto o rechazo agudo del injerto están asociados con una correlación negativa del componente físico de la CVRS 29,32. De tal manera, que a medida que disminuye el número de comorbilidades asociadas mejora la CVRS física ${ }^{22}$. Los ingresos hospitalarios y el número de días que permanecen ingresados presentan una correlación negativa y coeficientes bajos respecto a CVRS ${ }^{29}$.

Hipertensión y enfermedades cardiacas, en ocasiones como consecuencia de los efectos secundarios del tratamiento inmunosupresor, son las comorbilidades más comunes $\left(96^{\prime} 1 \%\right.$ y $31^{\prime} 2 \%$ ). La muerte en trasplante renal por enfermedades cardiacas supone un $38 \%{ }^{31}$.

\section{CVRS entre TR, HD, DP y población sana}

EITR presenta mejores puntuaciones de CVRS, cuando se compara con las dos técnicas de diálisis, HD y DP en las dimensiones del SF-36, así como en las variables de estrategias de afrontamiento y calidad de vida globa| $3,16,27,28,39,41,42,51,52,55,57$. Tan sólo un estudio documenta que la imagen corporal es peor el TR que en $\mathrm{HD}^{41}$.

Los receptores de trasplante renal refieren una CVRS próxima a la de la población general sana en igualdad de condiciones, pero sin llegar a alcanzar los valores medios. Por lo que siguen teniendo peores puntuaciones en CVRS que la población general16,42,43,45,51,52,55,59. Otro estudio refleja esto mismo, presentando el TR puntuaciones notablemente más bajas que la población general en siete de las ocho SF-36 escalas y las dos escalas de resumen, PCS y MCS. Las mayores diferencias se encontraban en la salud general, limitaciones de rol debido a las limitaciones de problemas de conducta y físicos debido a las escalas de problemas emocionales del SF-36 33 .

Sin embargo, en otras series publicadas las puntuaciones de los portadores de injerto renal funcionante se aproximan mucho a las de la media de población general y en algunos casos: funcionamiento social, rol emocional y salud mental son ligeramente superiores ${ }^{16}$. Incluso en alguna serie, los pacientes con TR tuvieron puntuaciones significativamente más altas en el funcionamiento físico, dolor corporal, salud general, y escala de funcionamiento social respecto a los pacientes en $\mathrm{HD}^{57}$.

En general, presentan CVRS en el componente físico, menor que la población general y similar en el componente mental ${ }^{12}$. 


\section{CVRS pre-post TR}

La CVRS mejora tras el TR con respecto al periodo pre-trasplante según varias publicaciones ${ }^{18,23,34,37,38,54}$. Las puntuaciones en el dominio físico variaron de 9'94 antes del trasplante a $17^{\prime} 41$ después del trasplante, con lo cual el trasplante reduce el impacto del dolor y la percepción de necesidad de tratamiento y mejora la energía, satisfacción del sueño, capacidad de caminar, de trabajar y de realizar las actividades básicas de la vida diaria. En cambio, otro estudio refleja que tras 12 meses post TR, la CVRS mejora en todas las dimensiones, tanto del componente físico como mental, salvo en la subescala de dolor donde los niveles son mayores en esta etapa con respecto a la anterior ${ }^{18,37}$. El aumento de vitalidad, la disminución de las restricciones físicas y síntomas así como la mejora de la disfunción cardiaca y actividades sociales son otros de los cambios que indican la mayor CVRS post $\mathrm{TR}^{26,38,54}$.

Respecto a la capacidad de trabajo el $76 \%$ de los receptores refieren no tener cambios, el $6 \%$ disminución de su capacidad y el $18 \%$ mejora de la capacidad de trabajo $0^{35}$.

\section{Cuestionarios para evaluar CVRS}

Se han utilizado diferentes cuestionarios para evaluar CVRS en el TR. De los 42 estudios analizados, veinticinco evaluaron la CVRS con el cuestionario genérico SF-36 $12,14,16,18,22,24,26,27,28,29,30,31,35,37,39,42,43,45,47,55,52,54,55,56,57$. En cambio, otros estudios han empleado otros cuestionarios para medir la CVRS como ReTransQol que mide las 5 dimensiones de salud física, salud mental, asistencia médica y satisfacción, tratamiento y temor a perder el injerto ${ }^{33}$. Otros autores han utilizado el Índice de Karnofsky que mide la capacidad para realizar actividades de la vida cotidiana con puntuaciones de 0-10032,35,38. Los cuestionarios MOS-SF 36 y SF-12, son versiones del SF-36 que evalúan las mismas 8 dimensiones de CVRS, pero de manera reducida y han sido utilizados en dos artículos ${ }^{33,22}$. EI WHOQOL-Bref mide 4 dimensiones: salud física, salud psicológica, relaciones sociales y ambiente ${ }^{34,50}$; el WHOQOL-100 evalúa 6 campos: físico, psicológico, independencia, relaciones sociales, ambiente y espiritualidad 336,41 y el MLDL mide las dimensiones físicas, psicológicas, situación social, vida diaria y índice global de calidad ${ }^{43}$. El EuroQol 5D mide las dimensiones de movilidad, cuidado personal, actividades cotidianas, dolor/malestar y ansiedad/depresión ${ }^{24}$.

Como cuestionarios más específicos se ha utilizado ESRD-SCL que evalúa la CVRS en receptores de TR teniendo en cuenta los efectos de la terapia inmunosupresora: limitaciones de capacidad física, capacidad cognitiva, disfunción cardiaca y renal, efectos secundarios de los esteroides, aumento del crecimiento del vello y trastornos psicológicos asociados al trasplante ${ }^{24}$; KDQOL-SF, cuestionario que utiliza 43 items específicos sobre la enfermedad renal y además incluye los 36 items del cuestionario SF-36, en este caso solo se evaluaron las 8 dimensiones del SF-3645; NIDDKQOL mide cinco ámbitos: síntomas físicos, estado psicológico (estrés emocional), función personal (capacidad y discapacidad de trabajo), función social, y percepción de la salud general58; TLC incluye demografía y elementos socioeconómicos, educación, tipo de seguro para cobertura médica y de farmacia, cuestiones específicas del trasplante incluida la duración en diálisis antes de el trasplante, el tiempo en lista de espera, trasplante de cadáver vs donante vivo, especialidad del médico, condiciones comórbidas, efectos adversos, uso de los esteroides y los índices de LSI (índice de satisfacción con la vida) y $T C I$ (índice de problemas en el cuidado del trasplante $)^{59}$.

Otros cuestionarios que se han analizado para evaluar la CVRS y obtener más datos acerca de factores que pudiesen influir en la misma son: $B S I^{43,51}, K-22^{40}, B f-S$ mide el estado de ánimo ${ }^{42}$.

\section{Discusión}

El objetivo que con esta revisión se pretendía era conocer los factores influyentes en la CVRS del paciente TR y determinar los cambios en la CVRS después del TR.

EITR se asocia con una mejora de la CVRS y un menor riesgo de mortalidad y disminución de los eventos cardiovasculares, si se compara con los diferentes tipos de TRS. EI TR es la modalidad preferida de tratamiento para la insuficiencia renal crónica, y justifica los intentos actuales para aumentar el número de pacientes en todo el mundo que se benefician de un trasplante de riñón. A pesar de la comorbilidad de los receptores de trasplante, los beneficios relativos del TR parecen estar aumentando con el tiempo, junto con la supervivencia frente a aquellos que aún se encuentran en lista de espera $^{60,61}$.

Tras la revisión de los distintos artículos se han observado relaciones con variables clínicas, psicológicas y sociodemográficas, pero aun así, no se tiene una respuesta clara de algunos factores que determinan la 
CVRS. Además, cada componente se puede expresar de diferentes maneras de acuerdo con la percepción subjetiva de cada paciente, resultando en una diferente evaluación de la calidad de vida. Por lo tanto, dos pacientes con condiciones clínicas y terapéuticas similares pueden percibir la calidad de vida de manera diferente, porque el concepto es el resultado de la interacción entre las condiciones de vida del paciente y la manera en que están percibidas ${ }^{62,63}$.

Los trastornos del sueño son muy comunes en los pacientes con ERC sometidos a TRS, siendo un probables predictor de mala CVRS en esta población. Este tipo de trastornos como el insomnio, aumentan con la edad y con la comorbilidad, presentando menor prevalencia entre los receptores de TR en comparación con los pacientes en diálisis ${ }^{64}$.

En cuanto al género, pese a las discrepancias encontradas, la mayoría de autores encuentran mejores puntuaciones en la CVRS en el sexo masculino en la mayoría de las dimensiones en comparación con las mujeres $29,30,31,34,52,59,65$.

Otro hallazgo llamativo fue la relación entre edad avanzada y un deterioro significativo del componente físico, pero no se asociaron predictores con afectación significativa de la calidad de vida mental $14,22,23,27,28,30,31,32,66$. Sin embargo el papel de la edad en la CVRS tiene enfoques controvertidos. En algunos estudios los pacientes con mayor edad presentan una CVRS más pobre, mientras en otros no se encontraron diferencias respecto a la edad o por el contrario si se hace referencia al papel global de la edad sobre la CVRS sin tener en cuenta el componente físico y mental, los jóvenes presentan peor CVRS frente a los receptores de edad avanza$\mathrm{da}^{3,37,39,430,45,52,59,62}$.

El estado civil y nivel de estudios han sido otras variables que han presentado cierta correlación positiva, asociándose el estar casado o vivir en pareja y el mayor nivel de estudios con mejor CVRS3,21,22,30,36,44,49,52,59.

Los datos bioquímicos son una de las variables más importantes a la hora de medir la CVRS, según se ha podido observar por su gran incidencia en la mayor parte de los artículos analizados donde se corroboran los mismos resultados. Los niveles elevados de hemoglobina y albúmina sérica junto con la disminución de creatinina sérica y aumento del hematocrito están asociados con mejor CVRS, mejorando la salud general, el funcionamiento físico y la vitali$\operatorname{dad}^{14,29,31,32,33,52,57,67 .}$
Otros factores como son el empleo y los ingresos influyen en la CVRS. De manera que el estar empleado y tener mayores ingresos implica mejores puntuaciones en el componente físico y mental $22,23,29,30,35,43,44,48,50,55$.

Los efectos secundarios del tratamiento inmunosupresor influyen negativamente en la CVRS de los pacientes con TR, debido a la presencia de modificaciones corporales principalmente, estrés, insomnio, cansancio, problemas gastrointestinales, dermatológicos y músculo esqueléticos ${ }^{12,23,27,30,46,59,68}$.

El tiempo post trasplante y previo en tratamiento de diálisis son factores con alto grado de influencia sobre la CVRS. Mayor tiempo en diálisis antes del TR disminuye la supervivencia post trasplante, la CVRS de componente físico y aumenta la ansiedad $30,31,39,51,55$. Tras el TR los destinatarios mejoran su CVRS respecto al periodo anterior y disminuyen el riesgo de mortalidad. Pese a ello, se han estudiado que existen periodos en los que algunos de los componentes físicos o mentales se encuentran disminuidos, regresando a las puntuaciones pre TR. No obstante, no se han obtenido datos relevantes sobre la mejora de CVRS a corto y largo plazo. Estas variables deberían ser estudiadas con mayor detenimiento $22,26,27,33,35,47,48,58,68$.

En cuanto al tipo de donante, los beneficiarios de trasplante renal procedente de donante vivo según informan varios autores, obtienen mejores puntuaciones en las escalas de funcionamiento físico en comparación con los receptores de injertos cadáver ${ }^{28,35,55,68}$.

La evolución y problemas psicológicos de los TR en función del tiempo trascurrido tras el trasplante y su influencia sobre la CVRS, se ha podido observar según los resultados de tres estudios que establecen periodos temporales de comparación. Menos de 5 años, entre 5 y 10 años y más de 10 años. Tienen peor calidad de vida los pacientes que llevan más tiempo trasplantados, probablemente, por ser de mayor edad y por los efectos secundarios de la medicación inmunosupresora47. A corto y a largo plazo hay una mejora de la calidad de vida, pero en el periodo intermedio (7-36 meses) los pacientes sufren un deterioro volviendo a los niveles pre-trasplante. Una posible explicación es que en la fase inmediatamente posterior al trasplante, los pacientes tienen un sentimiento de liberación con respecto a la dependencia de la diálisis, pero conforme pasa el tiempo han de afrontar diversos problemas somáticos (por ejemplo, los efectos secundarios de los medicamentos, las complicaciones médicas, etc.) y sociales (por ejemplo, la reincorporación laboral, la vida social, etc. $)^{48}$. Otro autor describe que los 
pacientes tienen más pensamientos negativos (ansiosos y depresivos) y peor autoestima física durante el primer año y a partir de los dos años de haber sido trasplantados, no existiendo diferencias significativas entre estos dos periodos, pero sí cuando se comparan ambos con el intervalo temporal 13-24 meses ${ }^{69}$.

En último lugar, en la revisión también aparecen los cambios en la CVRS antes y después del TR. La mejoría de los pacientes trasplantados con respecto al periodo anterior en HD ó DP es evidente y significativa. Diversos estudios han analizado la CVRS antes y después del TR y han documentado una mejoría en las facetas tanto físicas como mentales, así como menor necesidad de tratamiento, reducción al mínimo del impacto del dolor, mayor vitalidad al no depender de la diálisis y menores restricciones de movimientos, aunque se mantienen algunas limitaciones en el componente físico ${ }^{18,24,34,37,38,54}$.

\section{Conclusiones}

Se han dado respuesta en esta revisión a los objetivos planteados al principio de la misma, reflejándose a lo largo de la misma los factores asociados a la CVRS de los pacientes trasplantados de riñón y los cambios que llevan inmersos los beneficiarios de TR en el paso del periodo pre a post trasplante. Como factores fundamentales se encuentran factores sociodemográficos, médicos y psicológicos como son la edad, el sexo, los efectos secundarios del tratamiento inmunosupresor, el tiempo en diálisis, los problemas psicológicos y las comorbilidades.

Los factores que se han asociado con mejora de CVRS han sido el sexo masculino, estar casado o tener pare$\mathrm{ja}$, alto nivel de estudios, niveles elevados de hemoglobina y hematocrito, y trasplante renal procedente de donante vivo. Por el contrario existen una serie de factores que han presentado correlación negativa con CVRS como el sexo femenino, desempleo, elevados niveles de creatinina sérica, efectos secundarios del tratamiento inmunosupresor, presencia de comorbilidad asociada y mayor tiempo en TRS con diálisis antes de ser receptor de injerto renal.

Respecto a los cambios en la CVRS que tienen los beneficiarios de $T R$, en comparación con el periodo anterior en HD ó DP, se ha encontrado una mejora de la CVRS en todas las dimensiones. Sin embargo pese a esta mejora, las puntuaciones de los destinatarios de $\mathrm{TR}$, aunque se acercan a las de la población general de su misma edad y sexo, siguen sin ser alcanzadas.
Recibido: 20 julio 2015

Revisado: 25 julio 2015

Modificado: 31 julio 2015

Aceptado: 3 agosto 2015

\section{Bibliografía}

1. Soriano Cabrera S. Definición y clasificación de los estadios de la enfermedad renal crónica. Prevalencia. Claves para el diagnóstico precoz. Factores de riesgo de la enfermedad renal crónica. Nefrología. 2004; 24 Supl 6:S27-34.

2. Otero González A, De Francisco A, Gayoso P, García F. Prevalence of chronic renal disease in Spain: Results of the EPIRCE study. Nefrología. 2010; 30(1): 78-86.

3. Acosta Hernández PA, Chaparro López LC, Rey Anaconda CA. Quality of life and coping strategies in patients with chronic renal failure undregoing hemodialysis, peritoneal dialysis or kidney transplantation. Revista colombiana de psicología. 2008; (17): 9-26.

4. Malheiro Olivieira P, Arruda Soares D. Percepciones de las personas con insufiencia renal crónica sobre la calidad de vida. Enfermería global. 2012; 12 (1): 26-30.

5. Rebollo Rubio A, Morales Asensio JM, Pons Raventos MA, Mansilla Francisco JJ. Revisión de estudios sobre calidad de vida relacionada con la salud en la enfermedad renal crónica avanzada en España. Revista Nefrología. 2015;35: 92-109.

6. ALM de Francisco. El futuro del tratamiento de la enfermedad renal crónica. Nefrología. 2010;30(1): 1-9.

7. Registro Sociedad Española de Nefrología. Disponible en: http://www.senefro.org/modules/webstructure/files/inforreercongsen2014final.pdf. Revisado el 30-04-2015.

8. Registro European Dialysis and Trasplant Association. Disponible en: http://www.era-edta-reg.org/ index.jsp? $p=14$. Revisado el 30-04-2015. 
9. Fiebiger W, Mitterbauer C, Oberbauer R. Health-related quality of life outcomes after kidney transplantation. Health and Quality of Life Outcomes. 2004; doi:10.1186/1477-7525-2-2. Disponible en: http://www.ncbi.nlm.nih.gov/pmc/articles/ PMC317371/.

10. Dada Ruiz D, Fernández Aguirre C, Segura González F, Carballo Ruíz M. Indications and long term outcomes for solid organ transplant. Quality ol life in solid organ transplant recipients. Medicina Intensiva. 2008;32(6): 296-303.

11. García García G, Harden P, Chapman J. El papel global del trasplante. Nefrología. 2012:32(1): 1-6.

12. Liu H, Feurer ID, Dwyer K, Shaffer D \& Pinson CW. Effects of clinical factors on psychosocial variables in renal transplant recipients. Journal of Advanced Nursing. 2009;65(12),: 2585-2596.

13. Valentín M.0, Domínguez Gil B, Martín Escobar E, Matesanz R. No indicar el trasplante de vivo es una mala práctica. Nefrología. 2009;29(5): 379-381.

14. Borroto Díaz G, Almeida Fernández J, Lorenzo Clemente A, Alfonso Sat F, Guerreo Díaz C. Percepcion de la calidad de vida por enfermos sometidos a tratamientos de hemodiálisis o trasplante renal. Estudio comparativo. Revista cubana de medicina. 2007; 46(3). Disponible en: http:// scielo.sld.cu/scielo.php?script=sci_arttext\&pi$d=$ S0034-75232007000300004\&lng=es.

15. Humar A, Denny R, Matas AJ, Najarian JS. Graft and quality of life outcomes in older recipients of a kidney transplant. Exp Clin Transplant.2003;1(2):69-72.

16. Rebollo P, González M.P, Bobes J, Saiz P, Ortega F. Interpretación de los resultados de la calidad de vida relacionada con la salud de pacientes en terapia sustitutiva de la insuficiencia renal terminal. Nefrología. 2000:20(5): 431-439.

17. Ruiz Román $M^{a} J$, Román Cereto $M$, Martín Reyes G, Alférez Alférez Ma J, Prieto Merino D. Calidad de Vida Relacionada con la Salud en las diferentes terapias sustitutivas de la Insuficiencia Renal Crónica. .Rev Soc Esp Enferm Nefrol 2003; 6 (4):222-232.
18. Rodríguez Martín $M{ }^{a} C$, Conejos Alegre $C$, Fuenmayor Díaz A, Mirada, Ariet C, Sanz Izquierdo E, Torruella Barraquer C, Vela Ballestero A. Calidad de vida y trasplante renal en mayores de 65 años. Rev Soc Esp Enferm Nefrol 2009;12(1): 26-30.

19. Sreejitha NS, Devi KS, Deepa M, Narayana GL,Anil $M$, Rajesh R. The quality of life of patients in maintenance hemodialysis and those who underwent renal transplantation. Amrita J Med.2012;8: 1-44.

20. Chisholm-Burns MA, Erickson ER, Spivey CA, Gruessner R. WG, and Kaplan B. Concurrent validity of kidney transplant questionnaire in US renal transplant recipients. Patient Prefer Adherence. 2011;5: 517-522.

21. Pérez MA, Martín A, Gallego A, Santamaría JL. Influencia de algunas variables médicas y psicosociales en la recuperación psicológica de los trasplantados. Futuras líneas de intervención psicológica. Revista de psicopatología y psicología clínica. 2000;5(1): 77-87.

22. Prihodova L, Nagyova I, Rosenberger J, Roland R, Van Dijk JP and Groothoff JW. Impact of personality and psychological distress on health-related quality of life in kidney transplant recipients. Transplant International. 2010;23(5): 484-492.

23. Durán Muñoz M.I, Lope Andrea T, Del Pino Jurado $M^{a} \mathrm{R}$, Chicharro Chicharro $\mathrm{M}^{\mathrm{a}} \mathrm{C}$, Matilla Villar E. Percepción de la calidad de vida referida por el paciente adulto con trasplante renal. Enferm Nefrol. 2014;17(1): 45-50.

24. Pérez San Gregorio M. A, Martín Rodríguez A, Díaz Domínguez $\mathrm{R}$ y Pérez Bernal J. Evolución de la calidad de vida relacionada con la salud en los trasplantados renales. Nefrología. 2007;27(5):619626.

25. C. Valdés C y Ortega F. Avances en la calidad de vida relacionada con la salud y trasplante renal. Nefrología.2006;26(2):157-162.

26. Stømer U, Bergrem H, Gøransson LG. Health-related quality of life in kidney transplant patients and non-renal replacement therapy patients with chronic kidney disease stages 3b-4. Ann Transplant. 2013;18: 635-642. 
27. Czyżewski L, Sańko-Resmer J, Wyzgał J, Kurowski A. Assessment of Health-Related Quality of Life of Patients after Kidney Transplantation in Comparison with Hemodialysis and Peritoneal Dialysis. Ann Transplant. 2014;19: 576-585.

28. Balaska A, Moustafellos P, Gourgiotis S, Pistolas D, Hadjiyannakis E, Vougas V, Drakopoulos $S$. Changes in Health-Related Quality of Life in Greek Adult Patients 1 Year after Successful Renal Transplantation. Experimental and clinical transplantation. 2006;4(2):521-524.

29. Bohlke M, Marini SS, Rocha M, Terhorst L, Gomes $\mathrm{RH}$, Barcellos FC. et al. Factors associated with health-related quality of life after successful kidney transplantation: a population-based study. Qual Life Res.2009;18(9):1185-1193.

30. Gentile $E$, Beauger D, Speyer E, Jouve E, DussoL B, Jacquelinethttp://www.hqlo.com/content/11/1/88/ - ins 4 C and Briançon S. Factors associated with health-related quality of life in renal transplant recipients: results of a national survey in France. Health and Quality of Life Outcomes.2013; doi:10.1186/1477-7525-11-88. Disponible en: http://www.hqlo.com/content/11/1/88.

31. Rosenberger J, van Dijk J.P, Nagyova I, Roland R, Madarasova Geckova A, J. A. van den Heuvel W and W. Groothoff J. Do dialysis- and transplantation-related medical factors affect perceived health status?. Nephrol Dial Transplant. 2005;20: 2153-2158.

32. Arogundade F A, Abd-Essamie M A, Barsoum R S. Health-Related Quality of Life in Emotionally Related Kidney Transplantation: Deductions from a Comparative Study. Saudi J Kidney Dis Transpl. 2005;16(3): 11-20.

33. Wei TY, Chiang YJ, Hsieh CY, Weng LC, Lin SC, Lin $\mathrm{MH}$. Health related quality of life of long-term kidney transplantation recipients. Biomed J 2013;36: 243-251.

34. Mendonça Ana Elza Oliveira de, Salvetti Marina de Góes, Maia Eulália Maria Chaves, Silva Ana Cristina de Oliveira e, Torres Gilson de Vasconcelos. Analysis of the physical aspects of quality of life of kidney recipients. Rev. esc. enferm. 2015;49(1): 76-81.
35. Griva K, Stygall J, Ng JH, Davenport A, Harrison MJ, Newman S. Prospective Changes in Health-Related Quality of Life and Emotional Outcomes in Kidney Transplantation over 6 Years. Journal of Transplantation. 2011; doi:10.1155/2011/671571. Disponible en: http://www.ncbi.nlm.nih.gov/pmc/articles/PMC3142681/pdf/JTRAN2011-671571.pdf.

36. Ocampo Otálvaro LE, Zapata Rueda CM, Villa Londoño AJ y Vinaccia Alpi E. Calidad de vida relacionada con la salud en pacientes trasplantados de riñón, hígado y médula ósea Psicología y Salud: 2007;17 ( 1): 125-131.

37. Ortega $T$, Deulofeu $R$, Salamero $P$, Roman $A$, Masnou N, Rubio S, Garcia 0, Casanovas T, Cofán F, Twose J, Ortega F. Health-Related Quality of Life Before and After a Solid Organ Transplantation (Kidney, Liver, and Lung) of Four Catalonia Hospitals. Transplantation Proceedings. 2009;41: 2265-2267.

38. Canché-Arenas AP, Reza-Orozco M, RodríguezWeber FL. Calidad de vida en pacientes con trasplante renal del Hospital Ángeles del Pedregal. Med Int Mex. 2011;27(5): 446-454.

39. Rebollo P, Bobes J, González MP, Saiz P, Ortega F. Factores asociados a la calidad de vida relacionada con la salud (CVRS) de los pacientes en terapia renal sustitutiva (TRS). Nefrología.2000;20(2): 171-181.

40. Helga Franke G, Heemann U, Kohnle M, Luetkes P, Maehner N, Reimer J. Quality of life in patients before and after kidney transplantation. Psychology and Health. 2000;14: 1037-1049.

41. Tomasz W, Piotr S. A trial of objective comparison of quality between chronic renal failure patients treated with hemodialysis and renal transplantation. Ann Transplant. 2003;8: 47-53.

42. Maglakelidze N, Pantsulaia T, Tchokhonelidze I, Managadze L, and Chkhotua A. Assessment of Health-Related Quality of Life in Renal Transplant Recipients and Dialysis Patients. Transplantation Proceedings. 2011;43: 376-379.

43. Aasebø W, Agnete Homb-Vesteraas N, Hartmann A and Stavem K. Life situation and quality of life in young adult kidney transplant recipients. Nephrology Dialysis Transplantation.2008;24(1): 304-308. 
44. Yildirim A. The Importance of Patient Satisfaction and Health-Related Quality of Life after Renal Transplantation. Transplantation Proceedings. 2006;38: 2831-2834.

45. Costa-Requena G, Cantarell Aixendri MC, Rodriguez Urrutia A y Seron Micas D. Calidad de vida relacionada con la salud y trasplante renal: comparación con los valores poblacionales a los 6 meses postrasplante. Med Clin (Barc). 2014;142(9):393-396.

46. Ostrowski M, Wesołowski T, Makar D, Bohatyrewicz R. Changes in Patients' Quality of Life After Renal Transplantation. Transplantation Proceedings. 2000;32: 1371-1374.

47. Ichikawa $Y$, Fujisawa $M$, Hirose $E$, Kageyama $T$, Miyamoto Y, Sakai Y, Mori F, Isotani S, Yazawa K, Hanafusa T, Fujikubo M, Fukunishi T, Kamidono $S$ y Nagano S. Quality of life in kidney transplant patients. Transplantation Proceedings.2000;32, 1815-1816.

48. Ponto $P$, Rupolo GP, Marchini $F$, Feltrin $A$, Perin $\mathrm{N}$, Mazzoldi MA, Giacon B, Baldan N y Rigotti P. Quality of life change after kidney transplantation. Transplantation Proceedings.2001; 33， 18871889.

49. Akman B, 0 zdemir FN, Sezer S, Mic, ozkadiog lu $H$, and Haberal M. Depression Levels Before and After Renal Transplantation. Transplantation Proceedings. 2004;36: 111-113.

50. Lazzaretti CT, Carvalho JGR, Mulinari R.A, and Rasia JM. Kidney Transplantation Improves the Muldimensional Quality of Life. Transplantation Proceedings. 2004;36: 872-873.

51. Overbeck I, Bartels M, Decker 0, Harms J, Hauss J y Fangmann J. Changes in Quality of Life After Renal Transplantation. Transplantation Proceedings, 2005;37:1618-1621.

52. Ogutmen B, Yildirim A, Sever M.S, Bozfakioglu S, Ataman R, Erek E, Cetin 0, and Emel A. Health-Related Quality of Life After Kidney Transplantation in Comparison Intermittent Hemodialysis, Peritoneal Dialysis, and Normal Controls. Transplantation Proceedings. 2006;38: 419-421.

53. Shah V.S, Ananth A, Sohal G.K, Bertges-Yost W, Eshelman A, Parasuraman R.K, and Venkat K.K.
Quality of Life and Psychosocial Factors in Renal Transplant Recipients. Transplantation Proceedings.2006;38: 1283-1285.

54. Virzì $A$, Signorelli M.S, Veroux $M$, Giammarresi G, Maugeri S, Nicoletti A, and Veroux P. Depression and Quality of Life in Living Related Renal Transplantation. Transplantation Proceedings. 2007;39: 1791-1793.

55. Sayin A, Mutluay R, and Sindel S. Quality of Life in Hemodialysis, Peritoneal Dialysis, and Transplantation Patients. Transplantation Proceedings. 2007;39: 3047-3053.

56. Cornella C, Brustia M, Lazzarich E, Cofano F, Ceruso A, Barbé M.C, Fenoglio R, Cella $D$ and Stratta P. Quality of Life in Renal Transplant Patients Over 60 Years of Age. Transplantation Proceedings. 2008;40: 1865-1866.

57. Fujisawa M, Ichikawa $Y$, Yoshiya $K$, Isotani $S, H i-$ guchi A, Nagano S, Arakawa S, Hamami G, Matsumoto 0 , and Kamidono S. Assessment of Health-Related Quality of Life in Renal Transplant and Hemodialysis patients using the SF-36 health survey. Urology. 2000;56(2): 201-206.

58. Karam VH, Gasquet I, Delvart V, Hiesse C, Dorent $\mathrm{R}$, Danet C, Didier S, Charpentier B, Gandjbakhch I, Bismuto H, y Castaing D. Quality of life in adult survivors beyond 10 years after liver, kidney, and heart transplantation. Transplantation. 2003;76(12): 1699-1704.

59. Matas AJ, Halbert RJ, Barr ML, Helderman JH, Hricik DE, Pirsch JD, Schenkel FA, Siegal BR, Liu $H$, Ferguson RM. Life satisfaction and adverse effects in renal transplant recipients: a longitudinal analysis. Clin Transplant 2002;16: 113-121.

60. Tonelli $M$, Wiebe $N$, Knoll $G$, Bello A, Browne $S$, Jadhav $D$ et al. Systematic review: kidney transplantation compared with dialysis in clinically relevant outcomes. Am J Transplant. 2011;11(10):2093-2109.

61. Cassuto JR, Resse PP, Sonnad S, Bloom RD, Levine $\mathrm{MH}$, Olthoff KM, Shaked A, Naji A, Abt P. Wait list death and survival benefit of kidney transplantation among no renal transplant recipients. Am J Transplant. 2010;10: 2502- 2511. 
62. Fernando Valderrábanos, $M D, P h D$, Rosa Jofre, MD, and Juan M. López-Gómez, MD, PhD. Quality of Life in End-Stage Renal Disease Patients. American Journal of Kidney Diseases. 2001,38(3): 443-464.

63. Butt Z, Yount SE, Caicedo JC, Abecassis MM, Cella D. Quality of life assessment in renal transplant: review and future directions. Clin Transplant. 2008;22, 292-303.

64. Kovacs AZ, Molnar MZ, Szeifert $L$, Ambrus $C$, Molnar-Varga M, Szentkiralyi A. et al. Sleep disorders, depressive symptoms and health-related quality of life a cross-sectional comparison between kidney transplant recipients and waitlisted patients on maintenance dialysis. Nephrol Dial Transplant. 2011;26(3):1058-1065.

65. Beauger D, Gentile S, Jouve E, Dussol B, Jacquelinet $\mathrm{C}$, Briançon $\mathrm{S}$. Analysis, evaluation and adaptation of the ReTransQoL: a specific quality of life questionnaire for renal transplant recipients. Health and Quality of Life Outcomes. 2013; doi:10.1186/1477-7525-11-148. Disponible en: http://www.ncbi.nlm.nih.gov/pmc/articles/ PMC3766072/pdf/1477-7525-11-148.pdf.
66. Weber, Arman Faravardeh, Scott Jackson, Danielle Berglund, Richard Spong, Arthur J. Matas, Cynthia R. Gross, Hassan N. Ibrahim. Quality of life in elderly kidney transplant recipients. Journal of the American Geriatrics Society. 2014:62(10): 18771882.

67. Rebollo P, Ortega F, Ortega T, Valdés $C$, García-Mendoza M, Gómez E. Spanish validation of the "Kidney Transplant Questionnaire": a useful instrument for assessing health related quality of life in kidney transplant patients. Health and Quality of Life Outcomes. 2003;1: 56-65.

68. Huang $E$, Segev DL, Rabb H. Kidney transplantation in the elderly. Semin Nephrol.2009 Nov;29(6): 621-35.

69. Pérez MA., Martín A, Gallego A y Santamaría J.L. Influencia de algunas variables médicas y psicosociales en la recuperación psicológica de los trasplantados. Futuras líneas de intervención psicológica. Revista de Psicopatología y Psicología Clínica. 2000;5: 71- 87. 